\title{
Synthesis and Biological Evaluation of Novel Tricyclic Carbapenems (Trinems)
}

\author{
Osamu Kanno ${ }^{\dagger}$, Yasuo Shimoji ${ }^{\dagger}$, SATOSHI OHya $^{\dagger \dagger}$ and Isao KaWAmoto ${ }^{\dagger, *}$ \\ Medicinal Chemistry Research Laboratories ${ }^{\dagger}$ and Biological Research Laboratories, ${ }^{\dagger \dagger}$ Sankyo Co., Ltd. \\ 1-2-58 Hiromachi, Shinagawa-ku, Tokyo 140-8710, Japan
}

(Received for publication October 19, 1999)

\begin{abstract}
Synthesis of new tricyclic carbapenems (trinems) with a pyrrolidinyl moiety at the C-4 position of the tricyclic ring and their antimicrobial activities were studied. These trinems showed potent activities against Gram-positive bacteria including methicillin-resistant Staphylococcus aureus (MRSA). Among them, (4R)-[(S)-pyrrolidin-3-ylthiomethyl]trinem (14a) exhibited good activity against MRSA in vitro and in vivo.
\end{abstract}

Recently, MRSA has been causing serious problems in hospitals. ${ }^{1,2)}$ At present, worldwide, vancomycin is clinically the most popular treatment against MRSA. However, with the recent increased use of vancomycin, multiple-resistant Enterococcus faecium has emerged. In the field of carbapenem antibiotics, research to discover a new anti-MRSA agent to replace vancomycin continues today and these carbapenems are mostly $1 \beta$-methylcarbapenems. $^{3 \sim 6)}$ Recently, a novel class of tricyclic carbapenem (trinem) has been identified: sanfetrinem cilexetil (GV-118819, active form: sanfetrinem sodium, GV-104326) has been developed as an oral trinem by Glaxo SpA (Figure 1). ${ }^{7,8)}$ However, no trinems showing antiMRSA activity have been reported previously. Our attention was focused on the synthesis of novel trinems by introduction of a pyrrolidinyl moiety at the C-4 position. We synthesized two types of trinems with pyrrolidinylmethylthio or pyrrolidinylthiomethyl and pyrrolidinylmethylthiomethyl groups at the $\mathrm{C}-4$ position. Among them, $(4 R)$-[(S)-pyrrolidin-3-ylthiomethyl $]$ trinem (14a) exhibited potent anti-MRSA activity and good in vivo efficacy against experimental infection in mice compared with panipenem (PAPM), meropenem (MEPM), biapenem (BIPM) and vancomycin (VCM).

\section{Chemistry}

The two types of novel trinems were synthesized from Glaxo's epoxide intermediate $(\mathbf{1})^{9,10)}$ and 2hydroxymethylcyclohexanone intermediate $(6)^{11)}$ as shown in Schemes 1 and 2. The ring cleavage of 1 with $(S)$ - 3-acetylthiomethyl-1-allyloxycarbonylpyrrolidine in the presence of ethylenediamine followed by Swern oxidation afforded cyclohexanone $\mathbf{2 a}$ in $75 \%$ yield. Acylation of $\mathbf{2 a}$ with allyl oxalyl chloride and triethylamine afforded oxalamide $\mathbf{3 a}$ in $60 \%$ yield. The intramolecular Wittig type cyclization of $\mathbf{3 a}$ with diethyl ethylphosphonite in refluxing toluene for 4 hours gave protected trinem $4 \mathbf{a}$ in $36 \%$ yield. Deprotection of the $t$-butyldimethylsilyl (TBS) group of $\mathbf{4 a}$ with tetrabutylammonium fluoride (TBAF) followed by bis(triphenylphosphine)palladium dichloride and tributyltin hydride afforded trinem $\mathbf{5 a}$ in $58 \%$ yield ( 2 steps). Analogous reactions of 1 with $(R)$ 3-acetylthiomethyl-1-allyloxycarbonylpyrrolidine in the presence of ethylenediamine and $(S)$-1-allyloxycarbonyl2-mercaptomethylpyrrolidine afforded the corresponding ketones $\mathbf{2 b}$ and $\mathbf{2 c}$ in $68 \%$ and $70 \%$ yield, respectively. These ketones were acylated to give oxalamides $\mathbf{3 b}$ and $3 \mathrm{c}$ in $51 \%$ and $80 \%$ yield, respectively. The cyclization of

Fig. 1. Structure of sanfetrinem derivatives.

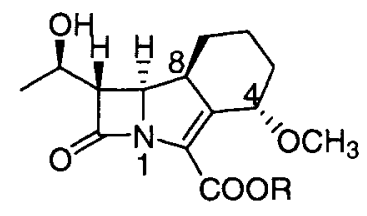

GV-104326 (sanfetrinem sodium) $R=\mathrm{Na}$

GV-118819 (sanfetrinem cilexetil) $R=$ 
Scheme 1.
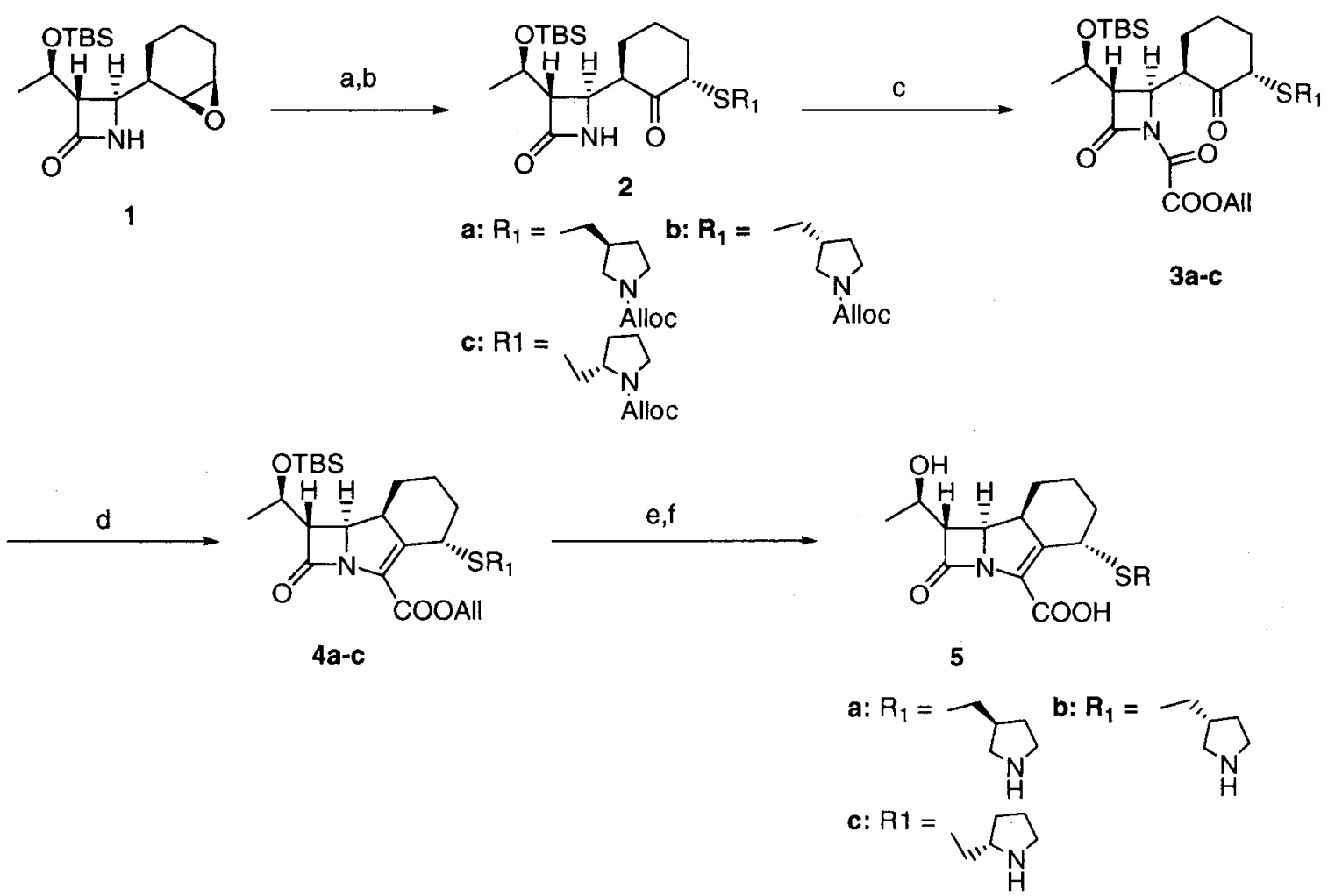

Reagents: (a) $\mathrm{R}_{1} \mathrm{SAc}, \mathrm{NH}_{2} \mathrm{CH}_{2} \mathrm{CH}_{2} \mathrm{NH}_{2}$ (2a, b) or $\mathrm{R}_{1} \mathrm{SH}, \mathrm{Et}_{3} \mathrm{~N}$ (2c); (b) Swern oxidation; (c) ClCOCOOAll, $\mathrm{Et}_{3} \mathrm{~N}$; (d) $\mathrm{EtP}(\mathrm{OEt})_{2}$; (e) TBAF or $\mathrm{HF} \cdot \mathrm{NH}_{4} \mathrm{~F}$; (f) $\mathrm{PdCl}_{2}\left(\mathrm{Ph}_{3} \mathrm{P}\right)_{2}, \mathrm{Bu}_{3} \mathrm{SnH}$.

3b with diethyl ethylphosphonite in refluxing toluene for 9 hours resulted in $16 \%$ yield, but that of $\mathbf{3 c}$ in refluxing toluene for 4 hours proceeded with $61 \%$ yield. These results in the cyclization reaction seem to reflect the steric hindrance of the $R_{1}$ group. Deprotection of both $4 b$ and $\mathbf{4 c}$ in a similar manner afforded trinems $\mathbf{5 b}$ and $\mathbf{5 c}$ in $46 \%$ and $36 \%$ yield, respectively.

Alternatively, thiomethyltrinem derivatives were synthesized from 2-hydroxymethylcyclohexanone derivative $\mathbf{6},{ }^{12}$ which was a versatile intermediate for the trinem synthesis. The mesylation of $\mathbf{6}$ with methanesulfonyl chloride and triethylamine afforded mesylate 7 in quantitative yield. Reaction of 7 with (S)-1-allyloxycarbonyl-3-mercaptopyrrolidine and (S)-1-allyloxycarbonyl-2-mercaptomethylpyrrolidine furnished trans- and cis-cyclohexanones $8 \mathrm{a}$ and $8 \mathrm{~b}$, and $9 \mathrm{a}$ and $9 \mathrm{~b}$, respectively. The ratios of $8 \mathbf{a}$ to $9 \mathbf{a}$ and $\mathbf{8 b}$ to $9 \mathbf{b}$ were both about $2: 3$. These reactions seem to proceeed via Michael addition to exo-methylene intermediate 10, because the conversion of $8 \mathbf{a}$ to $9 \mathrm{a}$ hardly occurred under the same conditions without thiol.
Acylation of $\mathbf{8 a}$ followed by cyclization with diethyl ethylphosphonite in refluxing toluene afforded protected trinem 11a in $81 \%$ yield. The stereochemistry at the C-4 position of 11a was confirmed by the observation of NOE between methylene protons of the side chain next to the $\mathrm{C}-4$ position and a proton at the C-8 position. ${ }^{12)}$ Deprotection of the TBS group of 11a followed by deallylation with bis(triphenylphosphine)palladium dichloride and tributyltin hydride afforded trinem 12a in 35\% yield ( 2 steps). On the other hand, acylation of 9a proceeded quantitatively, but cyclization of the oxalamide hardly occurred by refluxing in toluene due to the steric hindrance of the cis substituent groups of the cyclohexanone moiety. The cyclization occurred by refluxing in mesitylene for 2 hours to give trinem 13a in $66 \%$ yield. Deprotection of the TBS and allyl groups of 13a afforded trinem 14a in 36\% yield (2 steps). Analogous acylations of $\mathbf{8 b}$ and $\mathbf{9 b}$ followed by cyclizations in refluxing toluene furnished protected trinems 11b and 13b in $53 \%$ and $46 \%$, respectively ( 2 steps). This suggests that lower yields of $13 a$ and $13 b$ than those of $11 a$ and $11 b$ 
Scheme 2.

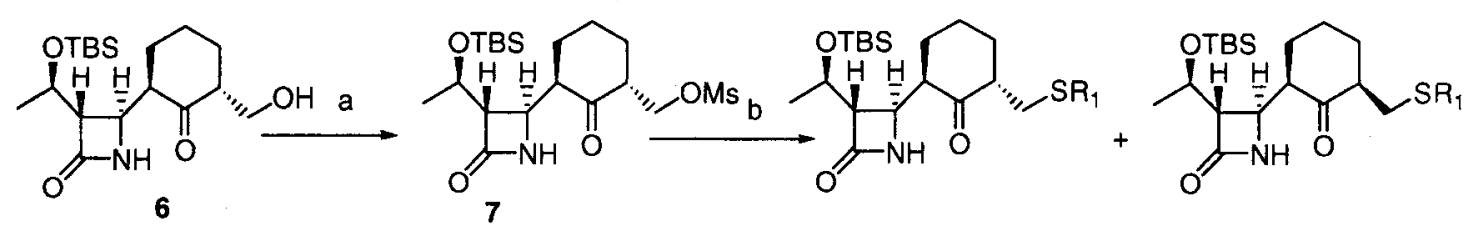<smiles>C=C1CCCC([C@H]2NC(=O)C3CCCC32C(C)C)C1=O</smiles>

8a: $R_{1}=$<smiles>CC1CCN(C)C1</smiles>

b: $R_{1}=$<smiles>CC[C@H]1CCCN1[N+](=O)[O-]</smiles>

9a: $R_{1}=$<smiles>CC1CN2CCN(C)C12</smiles>

b: $R_{1}=$<smiles>CC[C@H]1CCCN1C</smiles>

$8 a, b$

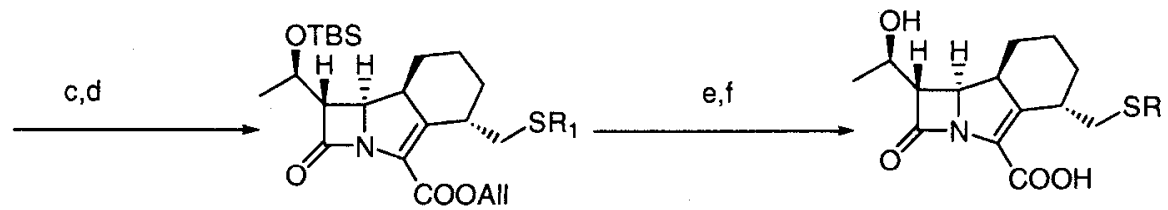

11a: $R_{1}=$<smiles>CC1CNC1</smiles>

b: $R_{1}=$

Álloc<smiles>CC[C@H]1CCCN1[Al]</smiles>

12a: $R=$<smiles>C[C@@H]1CCNC1</smiles>

b:<smiles>[2H]CC[C@H]1CCCN1</smiles>

9a,b

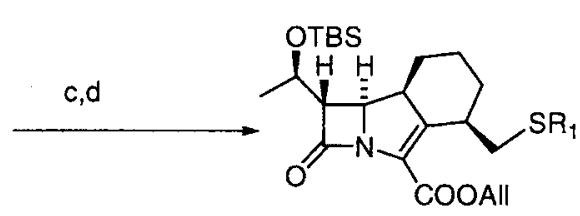

13a: $R_{1}=$<smiles>CC1CCNC1</smiles>

b: $R_{1}=$

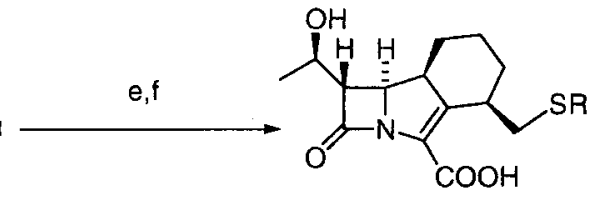

14a: $R=$<smiles>CC1CCNC1</smiles>

b: $R$<smiles>[CH2]C[C@H]1CCCN1</smiles>

Reagents: (a) $\mathrm{MsCl}, \mathrm{Et}_{3} \mathrm{~N}$; (b) $\mathrm{R}_{1} \mathrm{SH}, \mathrm{iPr}_{2} \mathrm{NEt}$; (c) ClCOCOOAll, $\mathrm{Et}_{3} \mathrm{~N}$; (d) $\mathrm{EtP}(\mathrm{OEt})_{2}$; (e) $\mathrm{HF} \cdot \mathrm{NH}_{4} \mathrm{~F}$ or TBAF; (f) $\mathrm{PdCl}_{2}\left(\mathrm{Ph}_{3} \mathrm{P}\right)_{2}, \mathrm{Bu}_{3} \mathrm{SnH}$.

were caused by steric bulkiness of the equatorial side chain. Deprotection of $11 \mathrm{~b}$ and $\mathbf{1 3 b}$ afforded trinems $12 \mathrm{~b}$ and $\mathbf{1 4 b}$ in $61 \%$ and $40 \%$, respectively ( 2 steps).

\section{Biological Properties}

The antibacterial activity (MICs) of trinems is shown in Table 1 . The trinems $5 \mathbf{a} \sim \mathbf{c}, \mathbf{1 2 a}, \mathbf{1 2 b}, \mathbf{1 4 a}$ and $\mathbf{1 4 b}$ showed potent activity against Gram-positive bacteria such as $S$. aureus 209P, but weak or moderate activity against Gramnegative bacteria such as $E$. coli NIHJ, $K$. pneumoniae 806 , and $S$. marcescens 1184. On the other hand, GV-104326 showed moderate activity against Gram-positive and Gramnegative bacteria, but weak activity against $S$. aureus 535 (MRSA). In spite of possessing an basic pyrrolidinyl moiety, these trinems which we synthesized rarely showed anti-pseudomonal activity with the exception of $\mathbf{5 a}$, which showed very weak activty against $P$. aeruginosa 1001 . The 
Table 1. Antibacterial activity (MIC, $\mu \mathrm{g} / \mathrm{ml})^{\mathrm{a}}$ of tricyclic carbapenems.

\begin{tabular}{|c|c|c|c|c|c|c|c|c|}
\hline Organism & $5 a$ & $\mathbf{5 b}$ & $5 c$ & $12 a$ & $12 b$ & $14 a$ & $14 b$ & GV-104326 \\
\hline $\begin{array}{l}\text { Staphylococcus aureus } 209 \mathrm{P} \\
\text { Staphylococcus aureus 56R } \\
\text { Staphylococcus aureus } 535 \text { (MRSA) } \\
\text { Enterococcus faecalis } 681 \\
\text { Escherichia coli NIHJ } \\
\text { Klebsiella pneumoniae } 806 \\
\text { Serratia marcescens } 1184 \\
\text { Pseudomonas aeruginosa } 1001\end{array}$ & $\begin{array}{l}\leq 0.01 \\
0.05 \\
3.1 \\
3.1 \\
6.2 \\
1.5 \\
6.2 \\
50\end{array}$ & $\begin{array}{l}\leq 0.01 \\
0.05 \\
6.2 \\
3.1 \\
12.5 \\
3.1 \\
12.5 \\
>50\end{array}$ & $\begin{array}{c}\leq 0.01 \\
0.05 \\
3.1 \\
1.5 \\
6.2 \\
1.5 \\
3.1 \\
>50\end{array}$ & $\begin{array}{l}0.02 \\
0.1 \\
12.5 \\
1.5 \\
0.8 \\
0.2 \\
0.4 \\
>50\end{array}$ & $\begin{array}{r}0.05 \\
0.1 \\
12.5 \\
1.5 \\
3.1 \\
0.8 \\
3.1 \\
>50\end{array}$ & $\begin{array}{l}0.02 \\
0.05 \\
1.5 \\
3.1 \\
1.5 \\
0.8 \\
3.1 \\
>50\end{array}$ & $\begin{array}{l}0.02 \\
0.1 \\
6.2 \\
6.2 \\
12.5 \\
3.1 \\
12.5 \\
>50\end{array}$ & $\begin{array}{c}0.02 \\
0.05 \\
12.5 \\
0.8 \\
0.2 \\
0.4 \\
0.8 \\
100\end{array}$ \\
\hline
\end{tabular}

${ }^{a} \mathrm{MIC}$ was determined by the agar dilution method with an inoculum of $10^{7} \mathrm{cfu} / \mathrm{ml}$.

Table 2. Protective effect of a tricyclic carbapenem 14a compared with those of PAPM, MEPM, BIPM and VCM against experimental infection in mice.

\begin{tabular}{|c|c|c|c|c|c|}
\hline \multirow{2}{*}{ Organism } & \multicolumn{5}{|c|}{$E_{50}(\mathrm{mg} / \mathrm{kg})^{\mathrm{a}}$} \\
\hline & $14 a$ & PAPM & MEPM & $\mathrm{BIPM}$ & $\mathrm{VCM}$ \\
\hline S. aureus $507^{\mathrm{b}}$ & 3.68 & 23.94 & 87.10 & 31.63 & 1.48 \\
\hline$[\mathrm{MIC}, \mu \mathrm{g} / \mathrm{ml}]^{\mathrm{c}}$ & {$[0.78]$} & {$[0.20]$} & [3.13] & {$[1.56]$} & {$[0.78]$} \\
\hline
\end{tabular}

${ }^{\text {a }} 50 \%$ effective sc dose.

${ }^{\mathrm{b}}$ Challenged with $5 \%$ mucin.

${ }^{\circ}$ Mueller-Hinton II agar was used as a medium.

activity of 4-thiotrinems $\mathbf{5 a} \sim \mathbf{c}$ against $S$. aureus 535 (MRSA) was compared with that of 4-thiomethyltrinems 12a, 12b, 14a and 14b. Among these trinems, 14a showed the most potent anti-MRSA activity and moderate activity against Gram-negative bacteria. The urinary recoveries of several trinems were measured after $\mathrm{sc}$ administration (50 $\mathrm{mg} / \mathrm{kg})$ in mice $(\mathrm{n}=5$, male, SPF $d d \mathrm{Y}$ strain). The urinary recoveries of $\mathbf{5 a}, 12 \mathbf{a}$ and $14 \mathbf{a}$ were $83 \%, 53 \%$ and $83 \%$, respectively. In order to clarify in vivo anti-MRSA activity, the protective effect of $14 a$ was compared to those of PAPM, MEPM, BIPM and VCM. The trinem 14a exhibited comparable in vivo efficacy to vancomycin against S. aureus 507 (MRSA, MIC against oxacillin: $32 \mu \mathrm{g} / \mathrm{ml}$ ). In vivo anti-MRSA efficacy of 14a was 6 23 times higher than those of PAPM, MEPM and BIPM. These results indicate a new possibility of trinem derivatives as potential anti-MRSA agents.

\section{Conclusion}

The structure-activity relationships of two types of tricyclic carbapenems (trinems) with a pyrrolidinyl moiety at the $\mathrm{C}-4$ position were clarified. The trinem 14a showed potent antimicrobial activity against Gram-positive bacteria including $S$. aureus 535 (MRSA) and higher in vivo efficacy against $S$. aureus 507 compared with those of panipenem, meropenem and biapenem. In vivo efficacy of 14a was comparable to that of vancomycin. Trinem 14a is of interest in the synthesis of potential anti-MRSA agents. 


\section{Experimental}

\section{General Methods}

IR spectra were recorded on a Nicolet NIC FT-IR (5SXC) spectrometer. NMR spectra were determined on a Jeol GX-270 $(270 \mathrm{MHz})$ or GX-400 $(400 \mathrm{MHz})$ spectrometer using tetramethylsilane (TMS) or sodium 3-(trimethylsilyl)-propionate- $d_{4}$ (TSP) as the internal standard. Mass spectra were recorded on JEOL HX-100, SX-102A or AX-505H mass spectrometer. The melting point (mp) was determined using a Yanagimoto micromelting point apparatus and was not corrected. Optical rotations were obtained with a Jasco DIP-370 polarimeter. UV spectra were recorded on a Shimadzu UV-3100 spectrometer. Column chromatography was carried out on a Silica gel $60(230 \sim 400 \mathrm{mesh}$, Art.9385, Merck) or a Cosmosil 75C $\mathrm{C}_{18}$ PREP (75 $\mu \mathrm{m}$, Nacalai Tesque, Inc.).

Synthesis of $(4 S, 8 S, 9 R, 10 S)$-10-[(R)-1-Hydroxyethyl]-11oxo-4-[(S)-pyrrolidin-3-ylmethylthio]-1-azatricyclo[7.2.0.0 $\left.0^{3,8}\right]$ undec-2-ene-2-carboxylic Acid (5a)

(1) $(3 S, 4 R)-4-[(2 S, 6 R)-2-[(S)-(1-$ Allyloxycarbonyl) pyrrolidin-3-ylmethylthio]cyclohexanon-6-yl]-3-[(R)-1(tert-butyldimethylsilyloxy)ethyl]azetidin-2-one (2a)

A solution of $(3 S, 4 R)-4-[(1 R, 2 S, 3 R)-2,3$-epoxycyclohexyl]3-[(R)-1-(tert-butyldimethylsilyloxy)ethyl]azetidin-2-one $(1,892 \mathrm{mg}, 2.74 \mathrm{mmol})$ and $(S)$-3-acetylthiomethyl-1-allyloxycarbonylpyrrolidine $(1.0 \mathrm{~g}, 4.11 \mathrm{mmol})$ in ethylenediamine $(2.74 \mathrm{ml}, 4.11 \mathrm{mmol})$ was stirred at $60^{\circ} \mathrm{C}$ for 2.5 hours. Ethyl acetate $(100 \mathrm{ml})$ was added to the reaction mixture and the mixture was washed with water, brine, dried over $\mathrm{Na}_{2} \mathrm{SO}_{4}$ and concentrated by evaporation under reduced pressure. The residue was purified by silica gel column chromatography (hexane-EtOAc, $1: 1$ ) to give $(3 S, 4 R)-4-[(1 S, 2 S, 6 R)-2-[(S)-(1-a 1 l y l o x y c a r b o n y l)-$ pyrrolidin-3-ylmethylthio]-1-hydroxycyclohexan-6-yl]-3[(R)-1-(tert-butyldimethylsilyloxy)ethyl $]$ azetidin-2-one $(1.07 \mathrm{~g}, 75 \%)$ as an oil: IR $(\mathrm{KBr}) \mathrm{cm}^{-1} 3424,3285,2931$, $2857,1740,1706,1685,1412 ;{ }^{1} \mathrm{H}$ NMR $\left(270 \mathrm{MHz}, \mathrm{CDCl}_{3}\right.$, TMS) $\delta 0.10(6 \mathrm{H}, \mathrm{s}), 0.89(9 \mathrm{H}, \mathrm{s}), 1.28(3 \mathrm{H}, \mathrm{d}, J=6.1 \mathrm{~Hz})$, $1.42 \sim 1.73(6 \mathrm{H}, \mathrm{m}), 1.95 \sim 2.13(2 \mathrm{H}, \mathrm{m}), 2.34 \sim 2.43(1 \mathrm{H}$, $\mathrm{m}), 2.52 \sim 2.65(2 \mathrm{H}, \mathrm{m}), 2.87 \sim 2.95(2 \mathrm{H}, \mathrm{m}), 3.07 \sim 3.20$ $(1 \mathrm{H}, \mathrm{m}), 3.34 \sim 3.48(1 \mathrm{H}, \mathrm{m}), 3.48 \sim 3.58(1 \mathrm{H}, \mathrm{m})$, $3.60 \sim 3.69(2 \mathrm{H}, \mathrm{m}), 3.92(1 \mathrm{H}, \mathrm{br} \mathrm{s}), 4.10 \sim 4.20(1 \mathrm{H}, \mathrm{m})$, $4.59(2 \mathrm{H}, \mathrm{d}, J=5.7 \mathrm{~Hz}), 5.21(1 \mathrm{H}, \mathrm{d}, J=10.1 \mathrm{~Hz}), 5.31(1 \mathrm{H}$, dd, $J=17.2,1.7 \mathrm{~Hz}), 5.88 \sim 6.02(2 \mathrm{H}, \mathrm{m})$. FAB-MS $m / z 527$ $(\mathrm{M}+\mathrm{H})^{+}$.

To a solution of oxalyl chloride $(0.44 \mathrm{ml}, 5.03 \mathrm{mmol})$ in $\mathrm{CH}_{2} \mathrm{Cl}_{2}(10 \mathrm{ml})$ was added dimethylsulfoxide $(0.71 \mathrm{ml}, 10.1$ mmol) dropwise at $-78^{\circ} \mathrm{C}$ under a dry nitrogen atomosphere. After stirring at $-78^{\circ} \mathrm{C}$ for 10 minutes, a solution of $(3 S, 4 R)-4-[(1 S, 2 S, 6 R)-2-[(S)-(1-a l l y l o x y c a r b o n y 1) p y r r o-$ lidin-3-ylmethylthio]-1-hydroxycyclohexan-6-yl]-3-[(R)-1(tert-butyldimethylsilyloxy)ethyl]azetidin-2-one $(1.06 \mathrm{~g}, 2.01$ $\mathrm{mmol})$ in $\mathrm{CH}_{2} \mathrm{Cl}_{2}(10 \mathrm{ml})$ was added to the mixture and the mixture was stirred at the same temperature for 10 minutes. Then, triethylamine $(2.8 \mathrm{ml}, 20 \mathrm{mmol})$ was added to the mixture followed by stirring at the same temperature for 30 minutes. The mixture was warmed to room temperature and EtOAc $(100 \mathrm{ml})$ was added thereto. The mixture was washed with water, brine, dried over $\mathrm{Na}_{2} \mathrm{SO}_{4}$ and concentrated by evaporation under reduced pressure. The residue was purified by silica gel column chromatography (hexane-EtOAc, $1: 1)$ to give $\mathbf{2 a}(1.02 \mathrm{~g}, 97 \%)$ as an oil: IR (neat) $\mathrm{cm}^{-1} 3277,2935,2858,1760,1704,1413,1106 ;{ }^{1} \mathrm{H}$ NMR $\left(270 \mathrm{MHz}, \mathrm{CDCl}_{3}, \mathrm{TMS}\right) \delta 0.07(3 \mathrm{H}, \mathrm{s}), 0.08(3 \mathrm{H}$, s), $0.88(9 \mathrm{H}, \mathrm{s}), 1.26(3 \mathrm{H}, \mathrm{d}, J=6.3 \mathrm{~Hz}), 1.70 \sim 2.21(8 \mathrm{H}$, m), $2.35 \sim 2.56(3 \mathrm{H}, \mathrm{m}), 2.90(1 \mathrm{H}, \mathrm{dd}, J=5.9,2.2 \mathrm{~Hz})$, $3.05 \sim 3.09(1 \mathrm{H}, \mathrm{m}), 3.33 \sim 3.68(5 \mathrm{H}, \mathrm{m}), 3.48 \sim 3.58(1 \mathrm{H}$, m), $4.08 \sim 4.23(2 \mathrm{H}, \mathrm{m}), 5.21(1 \mathrm{H}, \mathrm{d}, J=10.4 \mathrm{~Hz}), 5.30(1 \mathrm{H}$, dd, $J=17.2,1.3 \mathrm{~Hz}), 5.70(1 \mathrm{H}$, br d, $J=7.8 \mathrm{~Hz}), 5.89 \sim 6.00$ $(1 \mathrm{H}, \mathrm{m})$. FAB-MS $m / z 525(\mathrm{M}+\mathrm{H})^{+}$.

(2) $(3 S, 4 R)-1-$ Allyloxalyl-4-[(2S,6R)-2-[(S)-(1-allyloxycarbonyl)pyrrolidin-3-ylmethylthio]-cyclohexanon-6-yl]-3[(R)-1-(tert-butyldimethylsilyloxy)ethyl]azetidin-2-one (3a)

To a solution of $2 \mathrm{a}(600 \mathrm{mg}, 1.14 \mathrm{mmol})$ and triethylamine $(384 \mu \mathrm{l}, 2.74 \mathrm{mmol})$ in dichloromethane $(7 \mathrm{ml})$ was added dropwise allyloxalyl chloride ( $374 \mathrm{mg}, 2.52 \mathrm{mmol}$ ) in dichloromethane $(3 \mathrm{ml})$ under ice-cooling and the mixture was stirred for 30 minutes. 2-Propanol $(383 \mu 1,5.00 \mathrm{mmol})$ was added to the mixture and the mixture was concentrated under reduced pressure. The residue was purified by silica gel column chromatography (hexane -EtOAc, $3: 1$ ) to give 3a (438 mg, 60\%) as an oil: IR (neat) $\mathrm{cm}^{-1} 2933,2859$, $1808,1756,1704,1409,1215 ;{ }^{1} \mathrm{H}$ NMR $\left(270 \mathrm{MHz}, \mathrm{CDCl}_{3}\right.$, TMS) $\delta 0.04(3 \mathrm{H}, \mathrm{s}), 0.07(3 \mathrm{H}, \mathrm{s}), 0.85(9 \mathrm{H}, \mathrm{s}), 1.20(3 \mathrm{H}$, d. $J=6.4 \mathrm{~Hz}), \quad 1.44 \sim 1.80(8 \mathrm{H}, \mathrm{m}), 2.33 \sim 2.37(3 \mathrm{H}, \mathrm{m})$, $3.02 \sim 3.09(1 \mathrm{H}, \mathrm{m}), 3.28 \sim 3.64(5 \mathrm{H}, \mathrm{m}), 4.23 \sim 4.33(3 \mathrm{H}$, m), $4.58(2 \mathrm{H}, \mathrm{d}, J=5.4 \mathrm{~Hz}), 4.79(2 \mathrm{H}, \mathrm{d}, J=6.0 \mathrm{~Hz}), 5.18 \sim$ $5.43(4 \mathrm{H}, \mathrm{m}), 5.89 \sim 6.01(2 \mathrm{H}, \mathrm{m})$. FAB-MS $\mathrm{m} / \mathrm{z} 637$ $(\mathrm{M}+\mathrm{H})^{+}$.

(3) Allyl $(4 S, 8 S, 9 R, 10 S)-4-[(S)$-(1-allyloxycarbonyl)pyrrolidin-3-ylmethylthio]-10-[(R)-1-(tert-butyldimethylsilyloxy)ethyl]-11-oxo-1-azatricyclo[7,2.0.0 $\left.0^{3,8}\right]$ undec-2-ene-2carboxylate (4a)

A solution of $3 \mathrm{a}(423 \mathrm{mg}, 0.66 \mathrm{mmol})$ and diethyl ethylphosphonite $(299 \mathrm{mg}, 1.99 \mathrm{mmol})$ in dry toluene $(8 \mathrm{ml})$ was stirred under reflux for 4 hours. The mixture was concentrated under reduced pressure and the residue was 
purified by silica gel column chromatography (hexaneEtOAc, $4: 1)$ to give $4 \mathrm{a}(144 \mathrm{mg}, 36 \%)$ as an oil: IR (neat) $\mathrm{cm}^{-1} 2932,2858,1781,1709,1283,1196 ;{ }^{1} \mathrm{H}$ NMR $(270$ $\left.\mathrm{MHz}, \mathrm{CDCl}_{3}, \mathrm{TMS}\right) \delta 0.06(6 \mathrm{H}, \mathrm{s}), 0.89(9 \mathrm{H}, \mathrm{s}), 1.23(3 \mathrm{H}$, $\mathrm{d}, J=6.1 \mathrm{~Hz}), \quad 1.31 \sim 2.07(8 \mathrm{H}, \mathrm{m}), 2.37 \sim 2.56(3 \mathrm{H}, \mathrm{m})$, $3.03 \sim 3.12(1 \mathrm{H}, \mathrm{m}), 3.20(1 \mathrm{H}, \mathrm{d}, J=6.2,3.4 \mathrm{~Hz}), 3.32 \sim$ $3.70(4 \mathrm{H}, \mathrm{m}), 4.11 \sim 4.25(2 \mathrm{H}, \mathrm{m}), 4.59(2 \mathrm{H}, \mathrm{d}, J=5 \mathrm{~Hz})$, $4.62 \sim 4.80(2 \mathrm{H}, \mathrm{m}), 4.85(1 \mathrm{H}$, br s $), 5.18 \sim 5.47(4 \mathrm{H}, \mathrm{m})$, $5.88 \sim 6.04(2 \mathrm{H}, \mathrm{m})$. FAB-MS $m / z 605(\mathrm{M}+\mathrm{H})^{+}$.

(4) $(4 S, 8 S, 9 R, 10 S)-10-[(R)-1-H y d r o x y e t h y l]-11-o x o-4-$ $\left[(S)\right.$-pyrrolidin-3-ylmethylthio]-1-azatricyclo[7.2.0.0 $\left.0^{3,8}\right]$ undec-2-ene-2-carboxylic acid (5a)

To a solution of $4 \mathrm{a}(140 \mathrm{mg}, 0.23 \mathrm{mmol})$ in tetrahydrofuran $(3 \mathrm{ml})$ were added $1 \mathrm{M}$ tetrabutylammonium fluoride in tetrahydrofuran $(1.16 \mathrm{ml}, 1.16 \mathrm{mmol})$ and acetic acid $(80 \mu 1,1.39 \mathrm{mmol})$ under nitrogen atomosphere. The mixture was stirred at $0^{\circ} \mathrm{C}$ for 1 hour and left to stand in a refrigerator for 3 days. Ethyl acetate $(100 \mathrm{ml})$ was added to the mixture and the mixture was washed with brine, aqueous sodium hydrogencarbonate and dried over $\mathrm{Na}_{2} \mathrm{SO}_{4}$. The mixture was concentrated under reduced pressure and the residue was purified by silica gel column chromatography to afford allyl $(4 S, 8 S, 9 R, 10 S)-4-[(S)$-(1-allyloxycarbonyl)pyrrolidin-3-ylmethylthio]-10-[(R)-1-hydroxyethyl]-11-oxo-1-azatricyclo[7.2.0.0 $\left.{ }^{3,8}\right]$ undec-2-ene-2carboxylate ( $84 \mathrm{mg}, 74 \%$ ) as an oil: IR (neat) $\mathrm{cm}^{-1} 3436$, 2936, 1779, 1707, 1445, 11985, 1135; ' H NMR (270 MHz, $\left.\mathrm{CDCl}_{3}, \mathrm{TMS}\right) \delta 1.32(3 \mathrm{H}, \mathrm{d}, J=6.2 \mathrm{~Hz}), 1.27 \sim 2.05(8 \mathrm{H}$, $\mathrm{m}), 2.34 \sim 2.56(3 \mathrm{H}, \mathrm{m}), 3.02 \sim 3.09(1 \mathrm{H}, \mathrm{m}), 3.25(1 \mathrm{H}, \mathrm{dd}$, $J=6.5,3.5 \mathrm{~Hz}), 3.28 \sim 3.67(4 \mathrm{H}, \mathrm{m}), 4.21 \sim 4.32(2 \mathrm{H}, \mathrm{m})$, $4.59(2 \mathrm{H}, \mathrm{d}, J=5.3 \mathrm{~Hz}), 4.62 \sim 4.86(3 \mathrm{H}, \mathrm{m}), 5.18 \sim 5.47$ $(4 \mathrm{H}, \mathrm{m}), 5.88 \sim 6.04(2 \mathrm{H}, \mathrm{m})$. FAB-MS $m / z 491(\mathrm{M}+\mathrm{H})^{+}$.

To a solution of allyl $(4 S, 8 S, 9 R, 10 S)-4-[(S)$-(1-allyloxycarbonyl)pyrrolidin-3-ylmethylthio]-10-[(R)-1-hydroxyethyl]-11-oxo-1-azatricyclo[7.2.0.0 $0^{3,8}$ undec-2-ene-2-carboxylate $(81 \mathrm{mg}, 0.165 \mathrm{mmol})$ in dichloromethane $(1.6 \mathrm{ml})$ were added water $(16.4 \mu 1,0.91 \mathrm{mmol})$, bis(triphenylphosphine)palladium dichloride $(3.2 \mathrm{mg}, 0.0046 \mathrm{mmol})$ and tributyltin hydride $(222 \mu \mathrm{l}, 0.83 \mathrm{mmol})$ at $0 \sim 5^{\circ} \mathrm{C}$ under nitrogen atomosphere. The mixture was stirred at room temperature for 30 minutes. Dichloromethane $(20 \mathrm{ml})$ was added to the mixture and the mixture was extracted with water $(10 \mathrm{ml} \times 3)$. The aqueous layer was washed with dichloromethane and concentrated to $5 \mathrm{ml}$ under reduced pressure. The residue was purified by reversed phase column chromatography (Cosmosil $75 \mathrm{C}_{18}$ PREP, eluted with $3 \sim 9 \%$ acetonitrile-water). The desired fraction was concentrated under reduced pressure followed by lyophilization to give $\mathbf{5 a}(47 \mathrm{mg}, 78 \%)$ as a powder: IR $(\mathrm{KBr}) \mathrm{cm}^{-1} 3379,2930,1763,1587,1389 ;{ }^{1} \mathrm{H}$ NMR $(270$
$\left.\mathrm{MHz}, \mathrm{D}_{2} \mathrm{O}, \mathrm{TSP}\right) \delta 1.27(3 \mathrm{H}, \mathrm{d}, J=6.4 \mathrm{~Hz}), 1.36 \sim 1.45$ $(1 \mathrm{H}, \mathrm{m}), 1.67 \sim 1.85(3 \mathrm{H}, \mathrm{m}), 1.85 \sim 1.93 \quad(3 \mathrm{H}, \mathrm{m})$, $2.16 \sim 2.24(1 \mathrm{H}, \mathrm{m}), 2.50 \sim 2.63(3 \mathrm{H}, \mathrm{m}), 2.99(1 \mathrm{H}, \mathrm{dd}, J=$ $11.8,7.9 \mathrm{~Hz}), 3.27 \sim 3.55(6 \mathrm{H}, \mathrm{m}), 4.21(1 \mathrm{H}, \mathrm{dd}, J=10.6$, $3.3 \mathrm{~Hz}), 4.24(1 \mathrm{H}, \mathrm{q}, J=6.1 \mathrm{~Hz})$. FAB-MS $m / z 367(\mathrm{M}+$ $\mathrm{H})^{+}$.

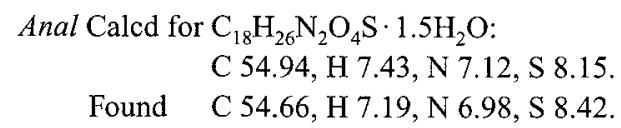

Synthesis of $(4 S, 8 S, 9 R, 10 S)-10-[(R)-1-$ Hydroxyethyl]-11oxo-4-[(R)-pyrrolidin-3-ylmethylthio]-1-azatricyclo[7.2.0.0 $\left.0^{3,8}\right]$ undec-2-ene-2-carboxylic Acid (5b)

(1) $(3 S, 4 R)-4-[(2 S, 6 R)-2-[(R)-(1-$ Allyloxycarbonyl)pyrrolidin-3-ylmethylthio]-cyclohexanon-6-yl]-3-[(R)-1-(tertbutyldimethylsilyloxy)ethyl]azetidin-2-one (2b)

The title compound $\mathbf{2 b}(470 \mathrm{mg}, 47 \%$ ) was prepared as an oil from $1(624 \mathrm{mg}, 1.92 \mathrm{mmol})$ by a similar manner as that described for the preparation of $2 \mathrm{a}$ : IR (neat) $\mathrm{cm}^{-1}$ $3275,2934,2858,1760,1704,1410,1106 ;{ }^{1} \mathrm{H}$ NMR $(270$ $\left.\mathrm{MHz}, \mathrm{CDCl}_{3}, \mathrm{TMS}\right) \delta 0.07(3 \mathrm{H}, \mathrm{s}), 0.08(3 \mathrm{H}, \mathrm{s}), 0.88(9 \mathrm{H}$, s), $1.26(3 \mathrm{H}, \mathrm{d}, J=6.2 \mathrm{~Hz}), 1.54 \sim 2.19(8 \mathrm{H}, \mathrm{m}), 2.34 \sim 2.52$ $(3 \mathrm{H}, \mathrm{m}), 2.90(1 \mathrm{H}, \mathrm{dd}, J=5.8,2.4 \mathrm{~Hz}), 3.05 \sim 3.10(1 \mathrm{H}, \mathrm{m})$, $3.32 \sim 3.62(5 \mathrm{H}, \mathrm{m}), 4.08 \sim 4.21(2 \mathrm{H}, \mathrm{m}), 4.59(2 \mathrm{H}, \mathrm{d}, J=$ $5.4 \mathrm{~Hz}), 5.23(1 \mathrm{H}, \mathrm{dd}, J=11.1,1.8 \mathrm{~Hz}), 5.31(1 \mathrm{H}, \mathrm{dd}, J=$ $3.2,1.3 \mathrm{~Hz}), 5.69(1 \mathrm{H}$, br s), $5.89 \sim 6.00(1 \mathrm{H}, \mathrm{m})$. FAB-MS $m / z 525(\mathrm{M}+\mathrm{H})^{+}$.

(2) $(3 S, 4 R)-1$-Allyloxalyl-4-[(2S,6R)-2-[(R)-(1-allyloxycarbonyl)pyrrolidin-3-ylmethylthio]-cyclohexanon-6-yl]-2oxocyclohexyl]-3-[(R)-1-(tert-butyldimethylsilyloxy)ethyl]azetidin-2-one (3b)

The title compound $\mathbf{3 b}(287 \mathrm{mg}, 51 \%)$ was prepared as an oil from $\mathbf{2 b}(460 \mathrm{mg}, 0.877 \mathrm{mmol}$ ) by a similar manner as that described for the preparation of $3 \mathbf{a}$ : IR (neat) $\mathrm{cm}^{-1}$ 2934, 2859, 1809, 1756, 1703, 1409, 1215; ${ }^{1} \mathrm{H}$ NMR (270 $\left.\mathrm{MHz}, \mathrm{CDCl}_{3}, \mathrm{TMS}\right) \delta 0.04(3 \mathrm{H}, \mathrm{s}), 0.07(3 \mathrm{H}, \mathrm{s}), 0.85(9 \mathrm{H}$, s), $1.20(3 \mathrm{H}, \mathrm{d}, J=6.4 \mathrm{~Hz}), 1.44 \sim 1.80(4 \mathrm{H}, \mathrm{m}), 1.97 \sim 2.17$ $(5 \mathrm{H}, \mathrm{m}), \quad 2.30 \sim 2.48(2 \mathrm{H}, \mathrm{m}), 3.00 \sim 3.08(1 \mathrm{H}, \mathrm{m})$, $3.30 \sim 3.39(3 \mathrm{H}, \mathrm{m}), 4.58(2 \mathrm{H}, \mathrm{d}, J=5.4 \mathrm{~Hz}), 4.76 \sim 4.80$ $(2 \mathrm{H}, \mathrm{m}), 5.13 \sim 5.46(4 \mathrm{H}, \mathrm{m}), 5.87 \sim 6.03(2 \mathrm{H}, \mathrm{m})$. FAB-MS $m / z 637(\mathrm{M}+\mathrm{H})^{+}$.

(3) Allyl $(4 S, 8 S, 9 R, 10 S)-4-[(R)$-(1-allyloxycarbonyl)pyrrolidin-3-ylmethylthio]-10-[(R)-1-(tert-butyldimethylsilyloxy)ethyl]-11-oxo-1-azatricyclo[7.2.0.0 $\left.0^{3,8}\right]$ undec-2ene-2-carboxylate (4b)

The title compound $4 \mathrm{~b}(42 \mathrm{mg}, 16 \%)$ was prepared as an oil from $\mathbf{3 b}(280 \mathrm{mg}, 0.44 \mathrm{mmol})$ by a similar manner as that described for the preparation of $4 \mathbf{a}$. IR (neat) $\mathrm{cm}^{-1}$ 2932, 2858, 1781, 1709, 1283, 1196; ' $\mathrm{H}$ NMR (270 MHz, $\left.\mathrm{CDCl}_{3}, \mathrm{TMS}\right) \delta 0.08(6 \mathrm{H}, \mathrm{s}), 0.89(9 \mathrm{H}, \mathrm{s}), 1.25(3 \mathrm{H}, \mathrm{d}, J=$ 
$6.1 \mathrm{~Hz}), 1.30 \sim 2.07(8 \mathrm{H}, \mathrm{m}), 2.37 \sim 2.56(3 \mathrm{H}, \mathrm{m}), 3.03 \sim$ $3.12(1 \mathrm{H}, \mathrm{m}), 3.22(1 \mathrm{H}, \mathrm{d}, J=6.3,3.4 \mathrm{~Hz}), 3.32 \sim 3.70(4 \mathrm{H}$, $\mathrm{m}), 4.13 \sim 4.26(2 \mathrm{H}, \mathrm{m}), 4.59(2 \mathrm{H}, \mathrm{d}, J=5.5 \mathrm{~Hz}), 4.60 \sim 4.87$ $(3 \mathrm{H}, \mathrm{m}), 5.18 \sim 5.47(4 \mathrm{H}, \mathrm{m}), 5.88 \sim 6.04(2 \mathrm{H}, \mathrm{m})$. FAB-MS $m / z 605(\mathrm{M}+\mathrm{H})^{+}$.

(4) $(4 S, 8 S, 9 R, 10 S)-10$-[(R)-1-Hydroxyethyl]-11-oxo-4$\left[(R)\right.$-pyrrolidin-3-ylmethylthio]-1-azatricyclo[7.2.0.0 $\left.0^{3,8}\right]$ undec-2-ene-2-carboxylic Acid (5b)

The title compound $\mathbf{5 b}(10 \mathrm{mg}, 46 \%)$ was prepared as a powder from $4 \mathbf{b}(40 \mathrm{mg}, 0.081 \mathrm{mmol})$ by a similar manner as that described for the preparation of 5a: IR $(\mathrm{KBr}) \mathrm{cm}^{-1}$ $3382,2929,1759,1600,1389 ;{ }^{1} \mathrm{H}$ NMR $\left(270 \mathrm{MHz}, \mathrm{D}_{2} \mathrm{O}\right.$, TSP) $\delta 1.27(3 \mathrm{H}, \mathrm{d}, J=6.4 \mathrm{~Hz}), 1.31 \sim 1.40(1 \mathrm{H}, \mathrm{m}), 1.67 \sim$ $1.97(6 \mathrm{H}, \mathrm{m}), 2.23 \sim 2.27(1 \mathrm{H}, \mathrm{m}), 2.54 \sim 2.60(3 \mathrm{H}, \mathrm{m})$, $2.93 \sim 3.01(3 \mathrm{H}, \mathrm{m}), 3.25 \sim 3.49(5 \mathrm{H}, \mathrm{m}), 4.21(1 \mathrm{H}, \mathrm{dd}, J=$ 9.5, 3.2 Hz), $4.25(1 \mathrm{H}, \mathrm{q}, J=6.3 \mathrm{~Hz})$. FAB-MS $m / z 367$ $(\mathrm{M}+\mathrm{H})^{+}$.

Synthesis of $(4 S, 8 S, 9 R, 10 S)-10-[(R)-1$-Hydroxyethyl]-11oxo-4-[(S)-pyrrolidin-2-ylmethylthio]-1-azatricyclo[7.2.0.0 $\left.0^{3,8}\right]$ undec-2-ene-2-carboxylic Acid (5c)

(1) $(3 S, 4 R)-4-[(2 S, 6 R)-2-[(S)$-(1-Allyloxycarbonyl)pyrrolidin-2-ylmethylthio]-cyclohexanon-6-yl]-3-[(R)-1-(tertbutyldimethylsilyloxy)ethyl]azetidin-2-one (2c)

To a solution of $1(1.46 \mathrm{~g}, 4.5 \mathrm{mmol})$ in methanol $(30 \mathrm{ml})$ were added dropwise triethylamine $(1.25 \mathrm{ml}, 8.92 \mathrm{mmol})$ and (S)-1-allyloxycarbonyl-2-mercaptomethylpyrrolidine $(1.36 \mathrm{~g}, 6.7 \mathrm{mmol})$ in methanol under ice-cooling and the mixture was stirred at room temperature for 2 days. Ethyl acetate $(150 \mathrm{ml})$ was added to the mixture and the mixture was washed with water, brine and dried over $\mathrm{Na}_{2} \mathrm{SO}_{4}$. After concentration of the mixture under reduced pressure, the residue was purified by silica gel column chromatography (Hexane-EtOAc, $1: 1)$ to give $(3 S, 4 R)-4-[(1 S, 2 S, 6 R)-2-$ [(S)-(1-allyloxycarbonyl)pyrrolidin-2-ylmethylthio]-1hydroxycyclohexan-6-yl]-3-[(R)-1-(tert-butyldimethylsilyloxy)ethyl]azetidin-2-one $(1.65 \mathrm{~g}, 70 \%)$ as an oil: IR $(\mathrm{KBr}) \mathrm{cm}^{-1} 3426,3285,2931,2857,1740,1705,1680$, 1412; ${ }^{1} \mathrm{H}$ NMR $\left(270 \mathrm{MHz}, \mathrm{CDCl}_{3}\right.$, TMS) $\delta 0.09(6 \mathrm{H}, \mathrm{s})$, $0.89(9 \mathrm{H}, \mathrm{s}), 1.27(3 \mathrm{H}, \mathrm{d}, J=5.6 \mathrm{~Hz}), 1.39 \sim 2.17(10 \mathrm{H}, \mathrm{m})$, $2.30 \sim 2.50(2 \mathrm{H}, \mathrm{m}), 2.94(1 \mathrm{H}, \mathrm{dd}, J=6.0,1.2 \mathrm{~Hz}), 2.79 \sim$ $3.14(2 \mathrm{H}, \mathrm{m}), 3.41 \sim 3.45(2 \mathrm{H}, \mathrm{m}), 3.65 \sim 3.69(1 \mathrm{H}, \mathrm{m})$, $3.94 \sim 4.08(2 \mathrm{H}, \mathrm{m}), 4.08 \sim 4.24(1 \mathrm{H}, \mathrm{m}), 4.58(2 \mathrm{H}, \mathrm{d}$, $J=5.1 \mathrm{~Hz}), 5.22(1 \mathrm{H}, \mathrm{dd}, J=10.1,1.8 \mathrm{~Hz}), 5.31(1 \mathrm{H}, \mathrm{d}, J=$ $18.6 \mathrm{~Hz}), 5.93(1 \mathrm{H}$, br s), $5.24 \sim 5.89(1 \mathrm{H}, \mathrm{m})$. FAB-MS $m / z$ $527(\mathrm{M}+\mathrm{H})^{+}$.

The title compound $2 \mathrm{c}(1.46 \mathrm{~g}, 89 \%)$ was prepared as an oil from $(3 S, 4 R)-4-[(1 S, 2 S, 6 R)-2-[(S)$-(1-allyloxycarbonyl) pyrrolidin-2-ylmethylthio]-1-hydroxycyclohexan-6-yl]-3[(R)-1-(tert-butyldimethylsilyloxy)ethyl]azetidin-2-one
$(1.64 \mathrm{~g}, 3.11 \mathrm{mmol})$ by a similar manner as that described for the preparation of $\mathbf{2 a}$ : IR $(\mathrm{KBr}) \mathrm{cm}^{-1} 3276,2952,2858$, 1758, 1704, 1406, 1105; ${ }^{\mathrm{H}} \mathrm{NMR}\left(270 \mathrm{MHz}, \mathrm{CDCl}_{3}, \mathrm{TMS}\right)$ $\delta 0.07(3 \mathrm{H}, \mathrm{s}), 0.08(3 \mathrm{H}, \mathrm{s}), 0.88(9 \mathrm{H}, \mathrm{s}), 1.26(3 \mathrm{H}, \mathrm{d}, J=$ $6.2 \mathrm{~Hz}), 1.44 \sim 2.21(10 \mathrm{H}, \mathrm{m}), 2.32 \sim 2.48(1 \mathrm{H}, \mathrm{m}), 2.76 \sim$ $2.85(1 \mathrm{H}, \mathrm{m}), 2.91(1 \mathrm{H}, \mathrm{dd}, J=5.3,3.6 \mathrm{~Hz}), 3.40 \sim 3.47(4 \mathrm{H}$, $\mathrm{m}), 3.94 \sim 3.99(1 \mathrm{H}, \mathrm{m}), 4.09(1 \mathrm{H}$, br s$), 4.10 \sim 4.23(1 \mathrm{H}$, $\mathrm{m}), 4.58 \sim 4.64(2 \mathrm{H}, \mathrm{m}), 5.20 \sim 5.34(2 \mathrm{H}, \mathrm{m}), 5.69(1 \mathrm{H}$, br $\mathrm{s}), 5.89 \sim 6.02(1 \mathrm{H}, \mathrm{m})$. FAB-MS $m / z 525(\mathrm{M}+\mathrm{H})^{+}$.

(2) $(3 S, 4 R)-1$-Allyloxalyl-4-[(2S,6R)-2-[(S)-(1-allyloxycarbonyl)pyrrolidin-2-ylmethylthio]-cyclohexanon-6-yl]-3[(R)-1-(tert-butyldimethylsilyloxy)ethyl]azetidin-2-one (3c)

The title compound $3 \mathrm{c}(1.39 \mathrm{~g}, 80 \%)$ was prepared as an oil from $2 \mathrm{c}(1.45 \mathrm{~g}, 2.76 \mathrm{mmol})$ by a similar manner as that described for the preparation of 3a: IR (neat) $\mathrm{cm}^{-1} 2952$, 2859, 1808, 1756, 1703, 1401, 1214; ${ }^{1} \mathrm{H}$ NMR (270 MHz, $\left.\mathrm{CDCl}_{3}, \mathrm{TMS}\right) \delta 0.03(3 \mathrm{H}, \mathrm{s}), 0.07(3 \mathrm{H}, \mathrm{s}), 0.85(9 \mathrm{H}, \mathrm{s})$, $1.22(3 \mathrm{H}, \mathrm{d}, J=7.3 \mathrm{~Hz}), 1.41 \sim 2.20(10 \mathrm{H}, \mathrm{m}), 2.24 \sim 2.41$ $(1 \mathrm{H}, \mathrm{m}), 2.71 \sim 2.91(1 \mathrm{H}, \mathrm{m}), 3.29(1 \mathrm{H}$, br s$), 3.35 \sim 3.44$ $(3 \mathrm{H}, \mathrm{m}), 3.88 \sim 4.11(1 \mathrm{H}, \mathrm{m}), 4.12 \sim 4.21(1 \mathrm{H}, \mathrm{m}), 4.26 \sim$ $4.35(2 \mathrm{H}, \mathrm{m}), 4.57 \sim 4.61(2 \mathrm{H}, \mathrm{m}), 4.78(2 \mathrm{H}, \mathrm{d}, J=6.0 \mathrm{~Hz})$, $5.18 \sim 5.88(4 \mathrm{H}, \mathrm{m}), 5.90 \sim 6.01(2 \mathrm{H}, \mathrm{m})$. FAB-MS $m / z 637$ $(\mathrm{M}+\mathrm{H})^{+}$.

(3) Allyl $(4 S, 8 S, 9 R, 10 S)-4-[(S)$-(1-allyloxycarbonyl)pyrrolidin-2-ylmethylthio $]-10-[(R)-1$-(tert-butyldimethylsilyloxy)ethyl]-11-oxo-1-azatricyclo[7.2.0.0 $\left.0^{3,8}\right]$ undec-2ene-2- carboxylate (4c)

The title compound $\mathbf{4 c}(808 \mathrm{mg}, 61 \%)$ was prepared as an oil from $3 \mathbf{c}(1.39 \mathrm{~g}, 2.18 \mathrm{mmol})$ by a similar manner as that described for the preparation of $4 \mathrm{a}$ : IR (neat) $\mathrm{cm}^{-1}$ 2931, 2857, 1781, 1706, 1403, 1284; ${ }^{1} \mathrm{H}$ NMR (270 MHz, $\left.\mathrm{CDCl}_{3}, \mathrm{TMS}\right) \delta 0.08(6 \mathrm{H}, \mathrm{s}), 0.89(9 \mathrm{H}, \mathrm{s}), 1.23(3 \mathrm{H}, \mathrm{d}, J=$ $6.1 \mathrm{~Hz}), 1.29 \sim 1.42(1 \mathrm{H}, \mathrm{m}), 1.68 \sim 2.05(9 \mathrm{H}, \mathrm{m}), 2.39 \sim$ $2.60(1 \mathrm{H}, \mathrm{m}), 2.89 \sim 2.95(1 \mathrm{H}, \mathrm{m}), 3.17(1 \mathrm{H}, \mathrm{dd}, J=6.1,3.1$ $\mathrm{Hz}), 3.34 \sim 3.51(3 \mathrm{H}, \mathrm{m}), 3.94 \sim 4.04(1 \mathrm{H}, \mathrm{m}), 4.08 \sim 4.25$ $(2 \mathrm{H}, \mathrm{m}), 4.57 \sim 4.83(4 \mathrm{H}, \mathrm{m}), 4.90(1 \mathrm{H}$, br s$), 5.17 \sim 5.47$ $(4 \mathrm{H}, \mathrm{m}), 5.88 \sim 6.01(2 \mathrm{H}, \mathrm{m})$. FAB-MS $m / z 605(\mathrm{M}+\mathrm{H})^{+}$.

(4) $(4 S, 8 S, 9 R, 10 S)-10-[(R)-1-H y d r o x y e t h y l]-11-o x o-4-$ $\left[(S)\right.$-pyrrolidin-2-ylmethylthio]-1-azatricyclo[7.2.0.0 $\left.0^{3,8}\right]$ undec-2-ene-2-carboxylic acid (5c)

To a solution of $\mathbf{4 c}(806 \mathrm{mg}, 1.33 \mathrm{mmol})$ in dimethylformamide $(5 \mathrm{ml})$ and $N$-methylpyrrolidone $(3.2 \mathrm{ml})$ was added ammonium hydrogenfluoride $(305 \mathrm{mg}, 5.3 \mathrm{mmol}$ ) at room temperature and the mixture was stirred at room temperature for 3 days. The mixture was treated and purified in the same manner as that described for the deprotection of the TBS group of $\mathbf{4 a}$ to give allyl $(4 S, 8 S, 9 R, 10 S)-4-[(S)$-(1-allyloxycarbonyl)pyrolidin-2ylmethylthio]-10-[(R)-1-hydroxyethyl]-11-oxo-1-azatricyclo[7.2.0.0 $\left.0^{3,8}\right]$ undec-2-ene-2-carboxylate $(447 \mathrm{mg}, 68 \%$ ) 
as an oil: IR (neat) $\mathrm{cm}^{-1} 3439,2936,1777,1705,1285$, 1195 ; ${ }^{1} \mathrm{H}$ NMR $\left(270 \mathrm{MHz}, \mathrm{CDCl}_{3}, \mathrm{TMS}\right) \delta 1.33(3 \mathrm{H}, \mathrm{d}, J=$ $6.1 \mathrm{~Hz}), 1.38 \sim 1.44(1 \mathrm{H}, \mathrm{m}), 1.68 \sim 2.04(9 \mathrm{H}, \mathrm{m}), 2.35 \sim$ $2.59(1 \mathrm{H}, \mathrm{m}), 2.90 \sim 3.01(1 \mathrm{H}, \mathrm{m}), 3.22(1 \mathrm{H}, \mathrm{dd}, J=6.4,3.1$ $\mathrm{Hz}), 3.35 \sim 3.52(3 \mathrm{H}, \mathrm{m}), 3.96 \sim 4.19(1 \mathrm{H}, \mathrm{m}), 4.20(1 \mathrm{H}, \mathrm{dd}$, $J=10.4,3.1 \mathrm{~Hz}), 4.24(1 \mathrm{H}, \mathrm{q}, J=6.2 \mathrm{~Hz}), 4.51 \sim 4.86(4 \mathrm{H}$, $\mathrm{m}), 4.89 \sim 4.91(1 \mathrm{H}, \mathrm{m}), 5.18 \sim 5.47(4 \mathrm{H}, \mathrm{m}), 5.87 \sim 6.03$ $(2 \mathrm{H}, \mathrm{m})$. FAB-MS $m / z 491(\mathrm{M}+\mathrm{H})^{+}$.

The title compound $5 \mathrm{c}(170 \mathrm{mg}, 53 \%)$ was prepared as a powder from allyl $(4 S, 8 S, 9 R, 10 S)-4-[(S)$-(1-allyloxycarbonyl)pyrrolidin-2-ylmethylthio]-10-[(R)-1-hydroxyethyl]11-oxo-1-azatricyclo[7.2.0.0 $\left.0^{3,8}\right]$ undec-2-ene-2-carboxylate (430 $\mathrm{mg}, 0.88 \mathrm{mmol}$ ) by a similar manner as that described for the preparation of $5 \mathrm{a}: \mathrm{IR}(\mathrm{KBr}) \mathrm{cm}^{-1} 3402,2930,1762$, $1587,1348,1215 ;{ }^{1} \mathrm{H}$ NMR $\left(270 \mathrm{MHz}, \mathrm{D}_{2} \mathrm{O}, \mathrm{TSP}\right) \delta 1.29$ $(3 \mathrm{H}, \mathrm{d}, J=6.6 \mathrm{~Hz}), 1.30 \sim 1.42(1 \mathrm{H}, \mathrm{m}), 1.76 \sim 2.14(10 \mathrm{H}$, $\mathrm{m}), 2.20 \sim 2.34(1 \mathrm{H}, \mathrm{m}), 2.70 \sim 2.90(1 \mathrm{H}, \mathrm{m}), 3.39(1 \mathrm{H}, \mathrm{dd}$, $J=11.8,6.6 \mathrm{~Hz}), 3.34 \sim 3.48(4 \mathrm{H}, \mathrm{m}), 3.73 \sim 3.78(1 \mathrm{H}, \mathrm{m})$, $4.21 \sim 4.29(2 \mathrm{H}, \mathrm{m}), 4.90(1 \mathrm{H}, \mathrm{br} \mathrm{s})$. FAB-MS $m / z 367$ $(\mathrm{M}+\mathrm{H})^{+}$.

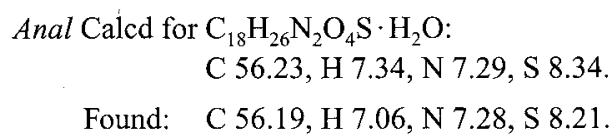

Synthesis of $(4 S, 8 S, 9 R, 10 S)-10-[(R)-1$-Hydroxyethyl]-4$[(S)$-pyrrolidin-3-ylthiomethyl]-11-oxo-1-azatricyclo[7.2.0.0 $\left.0^{3,8}\right]$ undec-2-ene-2-carboxylic Acid (12a)

(1) $(3 S, 4 R)-4-[(2 S, 6 R)-2-[(S)$-(1-Allyloxycarbonyl)pyrrolidin-3-ylthiomethyl $]$ cyclohexanon-6-yl]-3-[(R)-1-(tertbutyldimethylsilyloxy)ethyl]azetidin-2-one (8a) and $(3 S, 4 R)$ $4-[(2 R, 6 R)-2-[(S)$-(1-allyloxycarbonyl)pyrrolidin-3ylthiomethyl $]$ cyclohexanon-6-yl]-3-[(R)-1-(tertbutyldimethylsilyloxy)ethyl]azetidin-2-one (9a)

To a solution of $(3 S, 4 R)$-3-[(R)-1-(tert-butyldimethylsilyloxy)ethyl]-4-[(2R,6R)-2-(hydroxymethyl)cyclohexanon-2-yl]azetidin-2-one $(6,1.26 \mathrm{~g}, 3.54 \mathrm{mmol})$ in tetrahydrofuran $(15 \mathrm{ml})$ was added triethylamine $(596 \mu 1$, $4.25 \mathrm{mmol})$ and methanesulfonyl chloride $(302 \mu 1,3.90$ mmol) under ice-cooling. The mixture was stirred for 2 hours and the mixture was filtered. The filtrate was concentrated by evaporation under reduced pressure to give a mesylate. The mesylate was dissolved in dimethylformamide $(15 \mathrm{ml})$ and $(S)$-1-allyloxycarbonyl-3mercaptopyrrolidine $(1.33 \mathrm{~g}, 7.08 \mathrm{mmol})$ in dimethylformamide $(5 \mathrm{ml})$ and triethylamine $(596 \mu 1,4.25 \mathrm{mmol})$ was added to the mesylate solution. The mixture was stirred at room temperature for 2 hours and $40^{\circ} \mathrm{C}$ for 3 hours. The mixture was concentrated by evaporation under reduced pressure and the residue was purified by silica gel column chromatography (Hexane:AcOEt, 1:3) followed by preparative HPLC (cosmosil $5 \mathrm{C}_{18} \mathrm{AR} 28 \times 250 \mathrm{~mm}$ ) to give 8a (723 mg, 39\%) and 9a (1.03 g, 55\%) as colorless oils.

8a: IR $\left(\mathrm{CHCl}_{3}\right) \mathrm{cm}^{-1} 3417,2953,2860,1698,1413 ;{ }^{1} \mathrm{H}$ NMR $\left(400 \mathrm{MHz}, \mathrm{CDCl}_{3}, \mathrm{TMS}\right) \delta 0.06(3 \mathrm{H}, \mathrm{s}), 0.07(3 \mathrm{H}$, s), $0.87(9 \mathrm{H}, \mathrm{s}), 1.78(3 \mathrm{H}, \mathrm{d}, J=6.3 \mathrm{~Hz}), 1.58 \sim 2.26(8 \mathrm{H}$, $\mathrm{m}), 2.61 \sim 2.73(3 \mathrm{H}, \mathrm{m}), 2.85 \sim 2.92(2 \mathrm{H}, \mathrm{m}), 3.24 \sim 3.80$ $(5 \mathrm{H}, \mathrm{m}), 4.04(1 \mathrm{H}, \mathrm{dd}, J=5.7,1.7 \mathrm{~Hz}), 4.19(1 \mathrm{H}, \mathrm{qd}, J=6.3$, $5.1 \mathrm{~Hz}), 4.59(2 \mathrm{H}, \mathrm{d}, J=5.5 \mathrm{~Hz}), 5.21(1 \mathrm{H}, \mathrm{dd}, 10.3,1.3$ $\mathrm{Hz}), 5.31(1 \mathrm{H}, \mathrm{dd}, J=17.5,1.3 \mathrm{~Hz}), 5.85(1 \mathrm{H}$, br d, $J=6.0$ $\mathrm{Hz}), 5.89 \sim 5.99(1 \mathrm{H}, \mathrm{m})$. FAB-MS $m / z 525(\mathrm{M}+\mathrm{H})^{+}$.

9a: IR $\left(\mathrm{CHCl}_{3}\right) \mathrm{cm}^{-1} 3418,2953,2860,1753,1702$, 1412; ${ }^{1} \mathrm{H}$ NMR $\left(400 \mathrm{MHz}, \mathrm{CDCl}_{3}\right.$, TMS) $\delta 0.06(3 \mathrm{H}, \mathrm{s})$, $0.07(3 \mathrm{H}, \mathrm{s}), 0.87(9 \mathrm{H}, \mathrm{s}), 1.23(3 \mathrm{H}, \mathrm{d}, J=6.3 \mathrm{~Hz}), 1.33 \sim$ $2.62(11 \mathrm{H}, \mathrm{m}), 2.86(1 \mathrm{H}, \mathrm{dd}, J=4.8,2.4 \mathrm{~Hz}), 2.98(1 \mathrm{H}, \mathrm{dd}$, $J=13.9,5.8 \mathrm{~Hz}), 3.26 \sim 3.81(5 \mathrm{H}, \mathrm{m}), 4.09 \sim 4.12(1 \mathrm{H}, \mathrm{m})$, $4.20(1 \mathrm{H}, \mathrm{qd}, J=6.3,4.8 \mathrm{~Hz}), 4.59(2 \mathrm{H}, \mathrm{d}, J=5.9 \mathrm{~Hz}), 5.21$ $(1 \mathrm{H}, \mathrm{d}, J=10.3 \mathrm{~Hz}), 5.31(1 \mathrm{H}, \mathrm{d}, J=19.0 \mathrm{~Hz}), 5.72(1 \mathrm{H}, \mathrm{br}$ s), $5.89 \sim 5.99(1 \mathrm{H}, \mathrm{m})$. FAB-MS $m / z 525(\mathrm{M}+\mathrm{H})^{+}$.

(2) Allyl $(4 S, 8 S, 9 R, 10 S)$-4-[(S)-(1-allyloxycarbonyl)pyrrolidin-3-ylthiomethyl]-10-[(R)-1-(tert-butyldimethylsilyloxy)ethyl]-11-oxo-1-azatricyclo[7.2.0.0 $\left.0^{3,8}\right]$ undec-2ene-2-carboxylate (11a)

To a solution of $8 \mathbf{a} \quad(720 \mathrm{mg}, \quad 1.37 \mathrm{mmol})$ in dichloromethane $(10 \mathrm{ml})$ were added triethylamine $(384 \mu \mathrm{l}$, $2.74 \mathrm{mmol}$ ) and allyloxalyl chloride (305 $\mathrm{mg}, 2.06 \mathrm{mmol}$ ) under ice-cooling and the mixture was stirred for 1 hour. To the mixture was added 2-propanol $(52 \mu 1,0.69 \mathrm{mmol})$ and the mixture was stirred for 15 minutes. The mixture was concentrated by evaporation under reduced pressure and the residue was purified by silica gel column chromatography (hexane - AcOEt, $1: 1)$ to give $(3 S, 4 R)$-1-allyloxalyl-4-[(2S, $6 R)-2-[(S)$-(1-allyloxycarbonyl)pyrrolidin-3-ylthiomethyl]cyclohexanon-6-y1]-3-[(R)-1-(tert-butyldimethylsilyloxy)ethyl]azetidin-2-one $(873 \mathrm{mg}, 100 \%)$. To a solution of $(3 S, 4 R)-1$-allyloxalyl-4-[( $2 S, 6 R)-2-[(S)-(1$-allyloxycarbonyl)pyrrolidin-3-ylthiomethyl]-cyclohexanon-6-yl]-3[(R)-1-(tert-butyldimethylsilyloxy)ethyl]azetidin-2-one (873 $\mathrm{mg}, 1.37 \mathrm{mmol})$ in toluene $(2 \mathrm{ml})$ was added diethyl ethylphosphonite $(617 \mathrm{mg}, 4.11 \mathrm{mmol})$ and the mixture was stirred at $60^{\circ} \mathrm{C}$ for 1.5 hours. The mixture was concentrated by evaporation under reduced pressure and mesitylene $(50 \mathrm{ml})$ was added to the residue. The mixture was heated at $140^{\circ} \mathrm{C}$ for 1 hour and $120^{\circ} \mathrm{C}$ for 1.5 hours. The mixture was concentrated by evaporation under reduced pressure and the residue was purified by silica gel column chromatography (hexane-AcOEt, $3: 2$ to $1: 1$ ) to give 11a $(672 \mathrm{mg}, 81 \%)$ as an oil: $\mathrm{IR}\left(\mathrm{CHCl}_{3}\right) \mathrm{cm}^{-1} 2933$, $2860,1773,1693,1413 ;{ }^{1} \mathrm{H}$ NMR (400 MHz, $\left.\mathrm{CDCl}_{3}, \mathrm{TMS}\right)$ $\delta 0.07(3 \mathrm{H}, \mathrm{s}), 0.08(3 \mathrm{H}, \mathrm{s}), 0.88(9 \mathrm{H}, \mathrm{s}), 1.23(3 \mathrm{H}, \mathrm{d}, J=$ $6.4 \mathrm{~Hz}), 1.2 \sim 2.33(8 \mathrm{H}, \mathrm{m}), 2.68 \sim 2.80(2 \mathrm{H}, \mathrm{m}), 2.94(1 \mathrm{H}$, 
m), $3.17(1 \mathrm{H}, \mathrm{dd}, J=6.4,3.1 \mathrm{~Hz}), 3.24 \sim 3.62(4 \mathrm{H}, \mathrm{m})$, $3.75 \sim 3.80(1 \mathrm{H}, \mathrm{m}), 3.88(1 \mathrm{H}$, br s $), 4.11(1 \mathrm{H}, \mathrm{dd}, J=10.3$, $3.1 \mathrm{~Hz}), 4.20(1 \mathrm{H}, \mathrm{q}, J=6.4 \mathrm{~Hz}), 4.59(2 \mathrm{H}, \mathrm{d}, J=5.6 \mathrm{~Hz})$, $4.65 \sim 4.80(2 \mathrm{H}, \mathrm{m}), 5.21(1 \mathrm{H}, \mathrm{d}, J=13.2 \mathrm{~Hz}), 5.24(1 \mathrm{H}, \mathrm{d}$, $J=11.1 \mathrm{~Hz}), 5.31(1 \mathrm{H}, \mathrm{d}, J=17.5 \mathrm{~Hz}), 5.44(1 \mathrm{H}, \mathrm{d}, J=17.5$ $\mathrm{Hz}), 5.89 \sim 6.00(2 \mathrm{H}, \mathrm{m})$. FAB-MS $m / z 605(\mathrm{M}+\mathrm{H})^{+}$.

(3) $(4 S, 8 S, 9 R, 10 S)-10-[(R)-1-H y d r o x y e t h y l]-4-[(S)$ pyrrolidin-3-ylthiomethyl]-11-oxo-1-azatricyclo[7.2.0.0 $\left.0^{3,8}\right]$ undec-2-ene-2-carboxylic acid (12a)

To a solution of $11 \mathrm{a}(670 \mathrm{mg}, 1.11 \mathrm{mmol})$ in dimethylformamide $(10 \mathrm{ml})$ and $N$-methylpyrrolidone $(3.4 \mathrm{ml})$ was added ammonium hydrogenfluoride $(316 \mathrm{mg}, 5.54 \mathrm{mmol}$ ) at room temperature and the mixture was stirred at room temperature for 2 days. To the mixture was added 5\% aqueous $\mathrm{NaHCO}_{3}$ and then the mixture was extracted with AcOEt $(100 \mathrm{ml} \times 3)$. The extract was washed with brine, dried over $\mathrm{Na}_{2} \mathrm{SO}_{4}$ and concentrated by evaporation under reduced pressure. The residue was purified by silica gel column chromatography (hexane-AcOEt, 1:5) to give allyl $(4 S, 8 S, 9 R, 10 S)-4-[(S)$-(1-allyloxycarbonyl)pyrrolidin3-ylthiomethyl]-10-[(R)-1-hydroxyethyl]-1 1-oxo-1azatricyclo[7.2.0.0 $\left.0^{3,8}\right]$ undec-2-ene-2-carboxylate $(253 \mathrm{mg}$, 47\%): IR $\left(\mathrm{CHCl}_{3}\right) \mathrm{cm}^{-1} 3610,2940,1771,1693,1413 ;{ }^{1} \mathrm{H}$ NMR (400 MHz, CDCl 3 , TMS) $\delta 1.33(3 \mathrm{H}, \mathrm{d}, J=6.2 \mathrm{~Hz})$, $1.57 \sim 2.23(9 \mathrm{H}, \mathrm{m}), 2.69 \sim 2.81(2 \mathrm{H}, \mathrm{m}), 2.96 \sim 3.06(1 \mathrm{H}$, $\mathrm{m}), 3.22(1 \mathrm{H}, \mathrm{d}, J=6.6,3.1 \mathrm{~Hz}), 3.20 \sim 3.62(4 \mathrm{H}, \mathrm{m}), 3.77$ $(1 \mathrm{H}, \mathrm{dd}, J=10.6,6.0 \mathrm{~Hz}), 3.90(1 \mathrm{H}, \mathrm{m}), 4.17(1 \mathrm{H}, \mathrm{dd}$, $J=10.3,3.1 \mathrm{~Hz}), 4.21 \sim 4.28(1 \mathrm{H}, \mathrm{m}), 4.56(2 \mathrm{H}, \mathrm{d}, J=5.7$ $\mathrm{Hz}), 4.66 \sim 4.84(2 \mathrm{H}, \mathrm{m}), 5.21(1 \mathrm{H}, \mathrm{d}, J=10.1 \mathrm{~Hz}), 5.26$ $(1 \mathrm{H}, \mathrm{d}, J=10.1 \mathrm{~Hz}), 5.31(1 \mathrm{H}, \mathrm{d}, J=17.4 \mathrm{~Hz}), 5.44(1 \mathrm{H}, \mathrm{d}$, $J=17.4 \mathrm{~Hz}), \quad 5.89 \sim 6.02(1 \mathrm{H}, \quad \mathrm{m})$. FAB-MS $m / z \quad 491$ $(\mathrm{M}+\mathrm{H})^{+}$.

To a solution of allyl $(4 S, 8 S, 9 R, 10 S)-4-[(S)$-(1-allyloxycarbonyl)pyrrolidin-3-ylthiomethyl $]-10-[(R)$-1-hydroxyethyl]-11-oxo-1-azatricyclo[7.2.0.0 $\left.{ }^{3,8}\right]$ undec-2-ene-2carboxylate $(250 \mathrm{mg}, 0.52 \mathrm{mmol})$ in dichloromethane $(5.0$ $\mathrm{ml})$ were added water $(51 \mu \mathrm{l}, 2.84 \mathrm{mmol})$, bis(triphenylphosphine)palladium dichloride (18 $\mathrm{mg}, 0.026$ $\mathrm{mmol})$ and tributyltin hydride $(1.05 \mathrm{~g}, 3.61 \mathrm{mmol})$ at $0 \sim$ $5^{\circ} \mathrm{C}$ under nitrogen atomosphere. The mixture was stirred at room temperature for 30 minutes. Dichloromethane (30 $\mathrm{ml}$ ) was added to the mixture and the mixture was extracted with water $(30 \mathrm{ml} \times 3)$. The aqueous layer was washed with dichloromethane and concentrated to $5 \mathrm{ml}$ under reduced pressure. The residue was purified by reversed phase column chromatography (Cosmosil $75 \mathrm{C}_{18}$ PREP, eluted with $3 \sim 16 \%$ acetonitrile-water). The desired fraction was concentrated under reduced pressure followed by lyophilization to give $12 \mathbf{a}(127 \mathrm{mg}, 67 \%)$ as a colorless powder: IR $(\mathrm{KBr}) \mathrm{cm}^{-1} 3408,2929,1758,1586,1392$,
$1252 ;{ }^{1} \mathrm{H}$ NMR $\left(270 \mathrm{MHz}, \mathrm{D}_{2} \mathrm{O}, \mathrm{TSP}\right) \delta 1.27(3 \mathrm{H}, \mathrm{d}, J=6.2$ $\mathrm{Hz}), 1.26 \sim 1.38(1 \mathrm{H}, \mathrm{m}), 1.60 \sim 1.71(3 \mathrm{H}, \mathrm{m}), 1.82 \sim 2.00$ $(3 \mathrm{H}, \mathrm{m}), 2.34 \sim 2.44(1 \mathrm{H}, \mathrm{m}), 2.88(1 \mathrm{H}, \mathrm{d}, J=13.5,6.1 \mathrm{~Hz})$, $2.94(1 \mathrm{H}, \mathrm{d}, J=13.5,10.3 \mathrm{~Hz}), 2.97 \sim 3.06(1 \mathrm{H}, \mathrm{m}), 3.24$ $(1 \mathrm{H}, \mathrm{q}, J=6.8 \mathrm{~Hz}), 3.34 \sim 3.41(2 \mathrm{H}, \mathrm{m}), 3.49(1 \mathrm{H}, \mathrm{dt}, J=$ $11.8,7.5 \mathrm{~Hz}), 3.59 \sim 3.71(3 \mathrm{H}, \mathrm{m}), 4.15(1 \mathrm{H}$, dd, $J=10.7$, $3.0 \mathrm{~Hz}), 4.23(1 \mathrm{H}, \mathrm{q}, J=6.2 \mathrm{~Hz})$. FAB-MS $m / z 367$ $(\mathrm{M}+\mathrm{H})^{+}$.

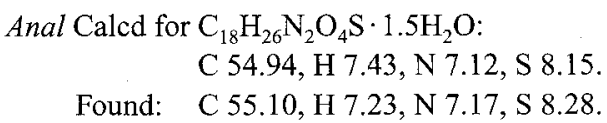

Synthesis of $(4 R, 8 S, 9 R, 10 S)-10-[(R)$-1-Hydroxyethyl]-4[ $(S)$-pyrrolidin-3-ylthiomethyl $]-11$-oxo-1-azatricyclo[7.2.0.0 $0^{3,8}$ undec-2-ene-2-carboxylic Acid (14a)

(1) Allyl $(4 R, 8 S, 9 R, 10 S)-4-[(S)$-(1-allyloxycarbonyl)pyrrolidin-3-ylthiomethyl]-10-[(R)-1-(tert-butyldimethylsilyloxy)ethyl]-11-oxo-1-azatricyclo[7.2.0.0 $\left.0^{3,8}\right]$ undec-2ene-2-carboxylate (13a)

To a solution of $9 \mathrm{a} \quad(460 \mathrm{mg}, \quad 0.88 \mathrm{mmol})$ in dichloromethane $(5 \mathrm{ml})$ were added triethylamine $(245 \mu \mathrm{l}$, $4.69 \mathrm{mmol}$ ) and allyloxalyl chloride (195 $\mathrm{mg}, 1.31 \mathrm{mmol})$ under ice-cooling and the mixture was stirred for 1.5 hours. To the mixture was added 2-propanol ( $33 \mu 1,0.44 \mathrm{mmol}$ ) and the mixture was stirred for 10 minutes. The mixture was concentrated by evaporation under reduced pressure and the residue was purified by silica gel column chromatography (hexane - AcOEt, $1: 1)$ to give $(3 S, 4 R)$-1allyloxalyl-4-[(2S, 6R)-2-[(S)-(1-allyloxycarbonyl)pyrrolidin3 -ylthiomethyl]cyclohexanon-6-yl]-3-[(R)-1-(tertbutyldimethylsilyloxy)ethyl]azetidin-2-one (556 $\mathrm{mg}, 99 \%)$. To a solution of $(3 S, 4 R)-1$-allyloxalyl-4-[(2S, 6R)-2-[(S)-(1allyloxycarbonyl)pyrrolidin-3-ylthiomethyl]-cyclohexanon6-yl]-3-[(R)-1-(tert-butyldimethylsilyloxy)ethyl]azetidin-2one $(556 \mathrm{mg}, 0.87 \mathrm{mmol})$ in toluene $(1 \mathrm{ml})$ was added diethyl ethylphosphonite $(655 \mathrm{mg}, 4.37 \mathrm{mmol})$ and the mixture was stirred at $60^{\circ} \mathrm{C}$ for 1.5 hours. The mixture was concentrated by evaporation under reduced pressure and mesitylene $(50 \mathrm{ml})$ was added to the residue. The mixture was heated at $130^{\circ} \mathrm{C}$ for 2.5 hours and refluxed for 2 hours. The mixture was concentrated by evaporation under reduced pressure and the residue was purified by silica gel column chromatography (hexane-AcOEt, $3: 1$ tol:1) to give 13a (348 $\mathrm{mg}, 66 \%)$ as an oil: IR $\left(\mathrm{CHCl}_{3}\right) \mathrm{cm}^{-1} 2933$, $1769,1691,1413 ;{ }^{\mathrm{l}} \mathrm{H} \mathrm{NMR}\left(400 \mathrm{MHz}, \mathrm{CDCl}_{3}, \mathrm{TMS}\right) \delta$ $0.07(6 \mathrm{H}, \mathrm{s}), 0.88(9 \mathrm{H}, \mathrm{s}), 1.22(3 \mathrm{H}, \mathrm{d}, J=6.2 \mathrm{~Hz})$, $1.17 \sim 1.61(3 \mathrm{H}, \mathrm{m}), 1.79 \sim 2.32(5 \mathrm{H}, \mathrm{m}), 2.53 \sim 2.82(3 \mathrm{H}$, m), $3.13 \sim 3.79(7 \mathrm{H}, \mathrm{m}), 4.09 \sim 4.22(2 \mathrm{H}, \mathrm{m}), 4.59(2 \mathrm{H}, \mathrm{d}$, $J=5.4 \mathrm{~Hz}), 4.62 \sim 4.80(2 \mathrm{H}, \mathrm{m}), 5.21(1 \mathrm{H}, \mathrm{d}, J=11.3 \mathrm{~Hz})$, $5.25(1 \mathrm{H}, \mathrm{d}, J=11.3 \mathrm{~Hz}), 5.30(1 \mathrm{H}, \mathrm{d}, J=17.0 \mathrm{~Hz}), 5.42$ 
$(1 \mathrm{H}, \mathrm{d}, J=17.0 \mathrm{~Hz}), 5.89(2 \mathrm{H}, \mathrm{m})$. FAB-MS $m / z 605$ $(\mathrm{M}+\mathrm{H})^{+}$.

(2) $(4 R, 8 S, 9 R, 10 S)-10-[(R)-1-H y d r o x y e t h y 1]-4-[(S)$ pyrrolidin-3-ylthiomethyl]-1 1-oxo-1-azatricyclo$\left[7.2 .0 .0^{3,8}\right]$ undec-2-ene-2- carboxylic acid (14a)

Allyl $\quad(4 R, 8 S, 9 R, 10 S)-4-[(S)$-(1-allyloxycarbonyl)pyrrolidin-3-ylthiomethyl $]-10-[(R)-1$-hydroxyethyl]-11-oxo-1azatricyclo[7.2.0.0 $\left.0^{3,8}\right]$ undec-2-ene-2-carboxylate $\quad(217 \mathrm{mg}$, $73 \%$ ) was prepared as an oil from 13a $(365 \mathrm{mg}, 0.60 \mathrm{mmol})$ by a similar manner as that described for the desilylation of 11a: ${ }^{1} \mathrm{H}$ NMR (400 MHz, $\mathrm{CDCl}_{3}$, TMS) $\delta 1.33(3 \mathrm{H}$, d, $J=6.3 \mathrm{~Hz}), 3.15 \sim 3.78(7 \mathrm{H}, \mathrm{m}), 4.11 \sim 4.24(2 \mathrm{H}, \mathrm{m}), 4.59$ $(2 \mathrm{H}, \mathrm{d}, J=5.4 \mathrm{~Hz}), 4.67 \sim 4.81(2 \mathrm{H}, \mathrm{m}), 5.19 \sim 5.49(4 \mathrm{H}, \mathrm{m})$, $5.87 \sim 6.06(2 \mathrm{H}, \mathrm{m})$. FAB-MS $m / z 491(\mathrm{M}+\mathrm{H})^{+}$.

The title compound 14a ( $80 \mathrm{mg}, 49 \%)$ was prepared as a powder from allyl $(4 R, 8 S, 9 R, 10 S)-4-[(S)$-(1-allyloxycarbonyl)pyrolidin-3-ylthiomethyl $]-10-[(R)-1$ hydroxyethyl]-11-oxo-1-azatricyclo[7.2.0.0 $\left.0^{3,8}\right]$ undec-2-ene2-carboxylate $(216 \mathrm{mg}, 0.441 \mathrm{mmol}$ ) by a similar manner as that described for the preparation of 12a: ${ }^{1} \mathrm{H}$ NMR (400 $\left.\mathrm{MHz}, \mathrm{D}_{2} \mathrm{O}, \mathrm{TSP}\right) \delta 1.08(3 \mathrm{H}, \mathrm{d}, J=6.4 \mathrm{~Hz}), 0.97 \sim 1.39$ $(3 \mathrm{H}, \mathrm{m}), 1.65 \sim 1.88(4 \mathrm{H}, \mathrm{m}), 2.15 \sim 2.24(1 \mathrm{H}, \mathrm{m}), 2.32 \sim$ $2.40(1 \mathrm{H}, \mathrm{m}), 2.58(1 \mathrm{H}, \mathrm{dd}, J=12.7,7.3 \mathrm{~Hz}), 2.62 \sim 2.68$ $(1 \mathrm{H}, \mathrm{m}), 3.00(1 \mathrm{H}, \mathrm{dd}, J=12.7,7.8 \mathrm{~Hz}), 3.05(1 \mathrm{H}, \mathrm{dd}$, $J=13.7,4.4 \mathrm{~Hz}), 3.14 \sim 3.22(2 \mathrm{H}, \mathrm{m}), 3.32(1 \mathrm{H}, \mathrm{td}, J=11.7$, $7.8 \mathrm{~Hz}), 3.38 \sim 3.48(2 \mathrm{H}, \mathrm{m}), 3.96(1 \mathrm{H}, \mathrm{dd}, J=9.8,2.9 \mathrm{~Hz})$, $4.03(\mathrm{q}, J=6.4 \mathrm{~Hz})$. FAB-MS $m / z 367(\mathrm{M}+\mathrm{H})^{+}$.

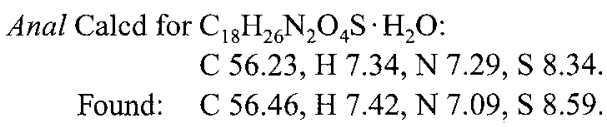

Synthesis of $(4 S, 8 S, 9 R, 10 S)-10-[(R)$-1-Hydroxyethyl]-4$[(S)$-pyrrolidin-2-ylmethylthiomethyl]-11-oxo-1-azatricyclo$\left[7.2 .0 .0^{3,8}\right]$ undec-2-ene-2-carboxylic Acid (12b)

(1) $(3 S, 4 R)-4-[(2 S, 6 R)-2-[(S)-(1-$ Allyloxycarbonyl)pyrrolidin-2-ylmethylthiomethyl]cyclohexanon-6-yl]-3-[(R)-1(tert-butyldimethylsilyloxy)ethyl]azetidin-2-one $(\mathbf{8 b})$ and

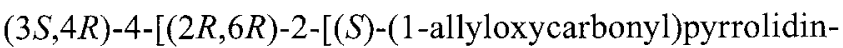
2-ylmethylthiomethyl $]$ cyclohexanon-6-yl]-3-[(R)-1-(tertbutyldimethylsilyloxy)ethyl]azetidin-2-one (9b)

The title compounds $\mathbf{8 b}$ (383 $\mathrm{mg}, 26 \%$ ) and $\mathbf{9 b}$ (543 mg, $37 \%$ ) were prepared as oils from $7(980 \mathrm{mg}, 2.76 \mathrm{mmol})$ by a similar manner as that described for the preparation of $\mathbf{8 a}$ and $9 \mathbf{a}$.

8b: $[\alpha]_{\mathrm{D}}^{25}=+13.4^{\circ}\left(c=0.70, \mathrm{CHCl}_{3}\right) ; \mathrm{IR}$ (neat) $\mathrm{cm}^{-1}$ 3276, 2932, 2858, 1761, 1705, 1406; ' $\mathrm{H}$ NMR (270 MHz, $\left.\mathrm{CDCl}_{3}, \mathrm{TMS}\right) \delta 0.06(3 \mathrm{H}, \mathrm{s}), 0.07(3 \mathrm{H}, \mathrm{s}), 0.87(9 \mathrm{H}, \mathrm{s})$, $1.20(3 \mathrm{H}, \mathrm{d}, J=6.2 \mathrm{~Hz}), 1.64 \sim 2.16(10 \mathrm{H}, \mathrm{m}), 2.40 \sim 3.44$ $(7 \mathrm{H}, \mathrm{m}), 3.38 \sim 3.44(2 \mathrm{H}, \mathrm{m}), 3.88 \sim 3.97(1 \mathrm{H}, \mathrm{m}), 4.02(1 \mathrm{H}$, $\mathrm{m}), 4.14 \sim 4.24(1 \mathrm{H}, \mathrm{m}), 4.57 \sim 4.62(2 \mathrm{H}, \mathrm{m}), 5.22(2 \mathrm{H}, \mathrm{d}$,
$J=10.5 \mathrm{~Hz}), 5.31(2 \mathrm{H}, \mathrm{d}, J=17.2 \mathrm{~Hz}), 5.87 \sim 6.02(1 \mathrm{H}, \mathrm{m})$, $6.23\left(1 \mathrm{H}\right.$, br s). FAB-MS $m / z 539(\mathrm{M}+\mathrm{H})^{+}$.

9b: $[\alpha]_{\mathrm{D}}^{25}=-4.4^{\circ}\left(c=0.85, \mathrm{CHCl}_{3}\right)$; IR (neat) $\mathrm{cm}^{-1} 3277$, $2931,2858,1760,1705,1405 ;{ }^{1} \mathrm{H}$ NMR $\left(270 \mathrm{MHz}, \mathrm{CDCl}_{3}\right.$, TMS) $\delta 0.06(3 \mathrm{H}, \mathrm{s}), 0.07(3 \mathrm{H}, \mathrm{s}), 0.87(9 \mathrm{H}, \mathrm{s}), 1.23(3 \mathrm{H}$, d, $J=6.0 \mathrm{~Hz}), 2.38 \sim 2.66(4 \mathrm{H}, \mathrm{m}), 2.82 \sim 3.04(2 \mathrm{H}, \mathrm{m}), 2.87$ (1H, dd, $J=4.8,2.4 \mathrm{~Hz}), 3.40 \sim 3.47(2 \mathrm{H}, \mathrm{m}), 3.92 \sim 3.98$ $(1 \mathrm{H}, \mathrm{m}), 4.08 \sim 4.22(2 \mathrm{H}, \mathrm{m}), 4.59(2 \mathrm{H}, \mathrm{m}), 5.21(1 \mathrm{H}, \mathrm{d}$, $J=8.8 \mathrm{~Hz}), 5.31(1 \mathrm{H}, \mathrm{d}, J=17.2 \mathrm{~Hz}), 5.75(1 \mathrm{H}$, br s $), 5.87 \sim$ $6.01(1 \mathrm{H}, \mathrm{m})$. FAB-MS $m / z 539(\mathrm{M}+\mathrm{H})^{+}$.

(2) Allyl $(4 S, 8 S, 9 R, 10 S)-4-[(S)$-(1-allyloxycarbonyl)pyrrolidin-2-ylmetylthiomethyl $]-10-[(R)$-1-(tert-butyldimethylsilyloxy)ethyl]-11-oxo-1-azatricyclo[7.2.0.0 $\left.0^{3,8}\right]$ undec-2-ene2-carboxylate (11b)

The title compound $11 \mathrm{~b}(200 \mathrm{mg}, 53 \%)$ was prepared as an oil from $8 \mathbf{b}(330 \mathrm{mg}, 0.612 \mathrm{mmol})$ by a similar manner as that described for the preparation of 11a: $[\alpha]_{\mathrm{D}}^{25}=+37.2^{\circ}$ $\left(c=0.79, \mathrm{CHCl}_{3}\right.$ ); IR (neat) $\mathrm{cm}^{-1} 2931,2858,1779,1702$, 1404; ${ }^{1} \mathrm{H}$ NMR $\left(270 \mathrm{MHz}, \mathrm{CDCl}_{3}, \mathrm{TMS}\right) \delta 0.08(6 \mathrm{H}, \mathrm{s})$, $0.88(9 \mathrm{H}, \mathrm{s}), 1.11 \sim 2.05(10 \mathrm{H}, \mathrm{m}), 1.23(3 \mathrm{H}, \mathrm{d}, J=6.3 \mathrm{~Hz})$, $2.37 \sim 2.54(1 \mathrm{H}, \mathrm{m}), 2.72 \sim 2.98(4 \mathrm{H}, \mathrm{m}), 3.16(1 \mathrm{H}, \mathrm{dd}, J=$ $6.4,3.1 \mathrm{~Hz}), 3.42 \sim 3.45(2 \mathrm{H}, \mathrm{m}), 3.84 \sim 4.00(2 \mathrm{H}, \mathrm{m}), 4.10$ $(1 \mathrm{H}, \mathrm{dd}, J=10.4,3.1 \mathrm{~Hz}), 4.20(1 \mathrm{H}, \mathrm{q}, J=6.3 \mathrm{~Hz}), 4.57 \sim$ $4.81(4 \mathrm{H}, \mathrm{m}), 5.18 \sim 5.46(4 \mathrm{H}, \mathrm{m}), 5.88 \sim 6.01(1 \mathrm{H}, \mathrm{m})$. FAB-MS $m / z 619(\mathrm{M}+\mathrm{H})^{+}$.

(3) $(4 S, 8 S, 9 R, 10 S)-10-[(R)-1-H y d r o x y e t h y l]-4-[(S)$-pyrrolidin-2-ylmethylthiomethyl]-11-oxo-1-azatricyclo[7.2.0.0 $\left.0^{3,8}\right]$ undec-2-ene-2- carboxylic acid (12b)

The title compound $\mathbf{1 2 b}(71 \mathrm{mg}, 61 \%)$ was prepared as a powder from $11 \mathrm{~b}(190 \mathrm{mg}, 0.31 \mathrm{mmol})$ by a similar manner as that described for the preparation of $12 \mathrm{a}$ : IR $(\mathrm{KBr}) \mathrm{cm}^{-1}$ $3372,2928,1758,1582,1390 ;{ }^{1} \mathrm{H}$ NMR $\left(400 \mathrm{MHz}, \mathrm{D}_{2} \mathrm{O}\right.$, TSP) $\delta 1.27(3 \mathrm{H}, \mathrm{d}, J=6.4 \mathrm{~Hz}), 1.28 \sim 1.38(1 \mathrm{H}, \mathrm{m}), 1.58 \sim$ $1.93(6 \mathrm{H}, \mathrm{m}), 1.99 \sim 2.16(2 \mathrm{H}, \mathrm{m}), 2.13 \sim 2.32(1 \mathrm{H}, \mathrm{m})$, $1.99 \sim 2.16(2 \mathrm{H}, \mathrm{m}), 2.13 \sim 2.32(1 \mathrm{H}, \mathrm{m}), 2.78 \sim 2.85(2 \mathrm{H}$, $\mathrm{m}), 2.94 \sim 3.01(2 \mathrm{H}, \mathrm{m}), 3.03 \sim 3.11(1 \mathrm{H}, \mathrm{m}), 3.29 \sim 3.38$ $(2 \mathrm{H}, \mathrm{m}), 3.40(1 \mathrm{H}, \mathrm{dd}, J=6.1,3.1 \mathrm{~Hz}), 3.66 \sim 3.72(1 \mathrm{H}, \mathrm{m})$, $3.74 \sim 3.83(1 \mathrm{H}, \mathrm{m}), 4.15(1 \mathrm{H}$, dd $J=10.2,3.1 \mathrm{~Hz}), 4.23$ $(1 \mathrm{H}, \mathrm{q}, J=6.4 \mathrm{~Hz})$. FAB-MS $m / z 381(\mathrm{M}+\mathrm{H})^{+}$.

$$
\begin{aligned}
& \text { Anal Caled for } \mathrm{C}_{19} \mathrm{H}_{28} \mathrm{~N}_{2} \mathrm{O}_{4} \mathrm{~S} \cdot 2 \mathrm{H}_{2} \mathrm{O} \text { : } \\
& \text { C 54.79, H 7.74, N 6.73, S 7.70. } \\
& \text { Found: C 55.81, H 7.65, N 6.72, S 7.52. }
\end{aligned}
$$

Synthesis of $(4 R, 8 S, 9 R, 10 S)-10-[(R)-1-$ Hydroxyethyl $]-4-$ [(S)-pyrrolidin-2-ylmethylthiomethyl]-11-oxo-1-azatricyclo$\underline{\left[7.2 .0 .0^{3,8}\right] \text { undec-2-ene-2-carboxylic Acid (14b) }}$

(1) Allyl $(4 R, 8 S, 9 R, 10 S)-4-[(S)$-(1-allyloxycarbonyl) pyrrolidin-2-ylmethylthiomethyl $]-10-[(R)$ - 1-(tert-butyldimethylsilyloxy)ethyl]-11-oxo-1-azatricyclo[7.2.0.0 $\left.0^{3,8}\right]$ undec-2-ene-2-carboxylate (13b) 
The title compound $13 \mathrm{~b}$ ( $265 \mathrm{mg}, 46 \%$ ) was prepared as an oil from $9 b(500 \mathrm{mg}, 0.93 \mathrm{mmol})$ by a similar manner as that described for the preparation of $13 \mathrm{a}:[\alpha]_{\mathrm{D}}^{25}=+54.5^{\circ}$ $\left(c=0.82, \mathrm{CHCl}_{3}\right) ; \mathrm{IR}$ (neat) $\mathrm{cm}^{-1} 2930,2856,1775$, 1701,$1405 ;{ }^{1} \mathrm{H}$ NMR $\left(270 \mathrm{MHz}, \mathrm{CDCl}_{3}, \mathrm{TMS}\right) \delta 0.07(6 \mathrm{H}$, s), $0.88(9 \mathrm{H}, \mathrm{s}), 1.11 \sim 2.96(10 \mathrm{H}, \mathrm{m}), 1.22(3 \mathrm{H}, \mathrm{d}, J=6.2$ $\mathrm{Hz}), 3.07 \sim 3.14(2 \mathrm{H}, \mathrm{m}), 3.42 \sim 3.43(2 \mathrm{H}, \mathrm{m}), 3.89 \sim 3.98$ $(1 \mathrm{H}, \mathrm{m}), 4.08 \sim 4.21(2 \mathrm{H}, \mathrm{m}), 4.57 \sim 4.81(4 \mathrm{H}, \mathrm{m}), 5.19 \sim$ $5.44(4 \mathrm{H}, \mathrm{m}), \quad 5.87 \sim 6.04(2 \mathrm{H}, \mathrm{m})$. FAB-MS $m / z 619$ $(\mathrm{M}+\mathrm{H})^{+}$.

(2) $(4 R, 8 S, 9 R, 10 S)-10-[(R)-1-H y d r o x y e t h y l]-4-[(S)$-pyrrolidin-2-ylmethylthiomethyl]-11-oxo-1-azatricyclo[7.2.0.0 $\left.0^{3,8}\right]$ undec-2-ene-2-carboxylic acid (14b)

The title compound $\mathbf{1 4 b}$ ( $59 \mathrm{mg}, 40 \%)$ was prepared as a powder from $13 \mathbf{b}(250 \mathrm{mg}, 0.404 \mathrm{mmol})$ by a similar manner as that described for the preparation of 12a: IR $(\mathrm{KBr}) \mathrm{cm}^{-1} 3363,2927,1758,1583,1390 ;{ }^{1} \mathrm{H}$ NMR (400 $\left.\mathrm{MHz}, \mathrm{D}_{2} \mathrm{O}, \mathrm{TSP}\right) \delta 1.23 \sim 1.37(1 \mathrm{H}, \mathrm{m}), 1.27(3 \mathrm{H}, \mathrm{d}, J=6.2$ $\mathrm{Hz}), 1.49 \sim 1.63(1 \mathrm{H}, \mathrm{m}), 1.68 \sim 1.78(1 \mathrm{H}, \mathrm{m}), 1.84 \sim 1.98$ $(3 \mathrm{H}, \mathrm{m}), 2.04 \sim 2.15(2 \mathrm{H}, \mathrm{m}), 2.22 \sim 2.30(1 \mathrm{H}, \mathrm{m}), 2.69(1 \mathrm{H}$, $\mathrm{dd}, J=11.8,5.7 \mathrm{~Hz}), 2.79 \sim 2.87(1 \mathrm{H}, \mathrm{m}), 2.76(1 \mathrm{H}, \mathrm{dd}, J=$ $14.6,10.0 \mathrm{~Hz}), 3.01(1 \mathrm{H}, \mathrm{dd}, J=14.6,4.5 \mathrm{~Hz}), 3.28(1 \mathrm{H}, \mathrm{dd}$, $J=11.8,9.5 \mathrm{~Hz}), 3.34 \sim 3.42(3 \mathrm{H}, \mathrm{m}), 3.79 \sim 3.87(1 \mathrm{H}, \mathrm{m})$, $4.15(1 \mathrm{H}, \mathrm{dd}, J=9.7,2.9 \mathrm{~Hz}), 4.22(1 \mathrm{H}, \mathrm{q}, J=6.2 \mathrm{~Hz})$. FAB$\mathrm{MS} m / z 381(\mathrm{M}+\mathrm{H})^{+}$.

Anal Calcd for $\mathrm{C}_{19} \mathrm{H}_{28} \mathrm{~N}_{2} \mathrm{O}_{4} \mathrm{~S} \cdot 2 \mathrm{H}_{2} \mathrm{O}$ : C 54.79, H 7.74, N 6.73, S 7.70.

Found: C 55.43, H 7.53, N 6.86, S 7.63.

\section{Measurement of Antibacterial Activity}

MICs were measured on Nutrient agar (Eiken Chemical Co., Ltd.) by the two-fold dilution method. The inoculum size of the bacteria was one-loopful of $10^{7} \mathrm{cfu} / \mathrm{ml}$.

\section{Therapeutic Effect on Systemic Infection in Mice}

Overnight cultures of $S$. aureus 507 grown at $37^{\circ} \mathrm{C}$ in Trypto-soy broth (Eiken Chemical Co., Ltd.) were diluted according to their virulence $\left(2.1 \times 10^{7} \mathrm{CFU} / \mathrm{mouse}\right)$. The diluted cultures were mixed with the same amount of $5 \%$ gastric mucin (Tokyo Kasei Kogyo Co., Ltd.). Seven male $\mathrm{SPF} d d \mathrm{Y}$ mice in each group were infected intraperitoneally with $0.2 \mathrm{ml}$ portions of these bacterial cultures. $\beta$ Lactam antibiotics (14a, PAPM, MEPM, BIPM) and vancomycin were administered subcutaneously at 0 and 4 hours after infection. The $\mathrm{ED}_{50}$ values of the mice were calculated by the probit method from the survival rates on the 5 th day after infection.
Acknowledgments

We wish to thank Mrs. S. KANEKo for her assistance with this synthetic work.

\section{References}

1) Chu, D. T. W.; J. J. Plattner \& L. Katz: New directions in antibacterial research. J. Med. Chem. 39: 3853 3874, 1996

2) Setti, E. L.; L. Quattrocchio \& R. G. Micetich: Current approaches to overcome bacterial resistance. Drugs Fut. 22: 271 284, 1997

3) Waddell, S. T.; R. W. Ratcliffe, S. P. Szumiloski, K. J. Kwildonger, R. R. Wilkening, T. A. Blizzard, J. Huber, J. Kohler, K. Dorso, E. S. Rose, J. G. Sundelof \& G. G. HAMmond: Benzothiazolylthio carbapenems: Potent anti-MRSA agents. Bioorg. Med. Chem. Lett. 5: 1427 1432, 1995

4) Sumita, Y; H, Nouda, K. Kanazawa \& M. Fukasawa: Antimicrobial activity of SM-17466, a novel carbapenem antibiotic with potent activity against methicillinresistant Staphylococcus aureus. Antimicrob. Agents Chemother. 39: 910 916, 1995

5) Otake, N.; H. Imamura, H. Kiyona, H, Jona, M. Ogawa, S. Okada, A. Shimizu, M. Moriya, Y. Tominaga, K. Yamada, M. Nakano, R. UshiJima \& S. NAKAGAWA: Novel dithiocarbamate carbapenems with anti-MRSA activity. Bioorg. Med. Chem. Lett. 7: $1617 \sim 1622,1997$

6) Кашамото, I.: $1 \beta$-Methylcarbapenem antibiotics. Drugs Fut. 23: $181 \sim 189,1998$

7) Gaviraghi, G.: Tribactams: A new prospect in $\beta$-lactam chemotherapy. Eur. J. Med. Chem. 30 (Suppl.) 467s 478s, 1995

8) Di Modugno, E.; I. Erbetti, L. Ferrari, G. Glassi, S. M. HAMmOND \& L. XERRI: In vitro activity of the tribactam GV104326 against Gram-positive, Gramnegative, and anaerobic bacteria. Antimicrob. Agents Chemother. 38: 2362 2368, 1994

9) Rossi, T.; S. Biondi, S. Contini, R. J. Thomas \& C. MARCHIORO: Novel amidoalkylation of 4-acetoxyazetidinones with allylic boranes. A stereoselective entry into the tribactams. J. Am. Chem. Soc. 117: 9604 9605, 1995

10) Ghiron, C.; E. Piga, T. Rossi, B. Tamburini \& R. J. THOMAs: The stereoselective synthesis of a key intermediate of the trinem antibiotic sanfetrinem. Tetrahedron Lett. 36: 4283, 1995

11) Kawamoto, I.; O. Kanno, S. Kobayashi, Y. Shimoj \& S. OHYA: New heterocyclic derivatives: Useful for the treatment and prevention of bacterial infection. PCT Internat. Appl. WO9834937-Al (8/13/1998)

12) KanNo, O. \& I. KaWAMOTO: Stereoselective synthesis of novel anti-MRSA tricyclic carbapenems (trinems). Tetrahedron, in press. 